Document downloaded from:

http://hdl.handle.net/10251/154387

This paper must be cited as:

Payri, F.; García-Oliver, JM.; Novella Rosa, R.; Pérez-Sánchez, EJ. (2019). Influence of the $\mathrm{n}$-dodecane chemical mechanism on the CFD modelling of the diesel-like ECN Spray A flame structure at different ambient conditions. Combustion and Flame. 208:198-218. https://doi.org/10.1016/j.combustflame.2019.06.032

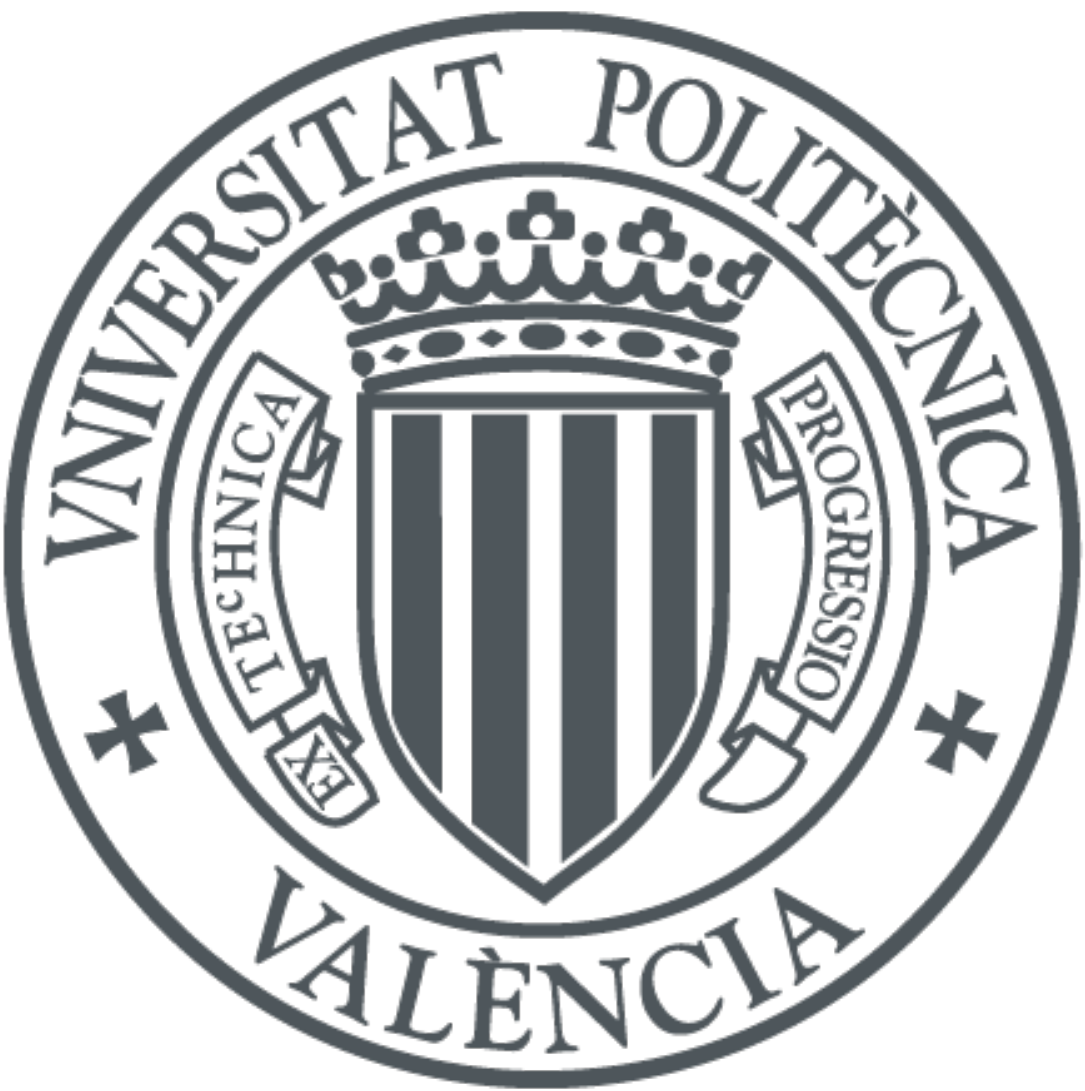

The final publication is available at

https://doi.org/10.1016/j.combustflame.2019.06.032

Copyright Elsevier

Additional Information 


\title{
Influence of the n-dodecane chemical mechanism on the CFD modelling of the diesel-like ECN Spray A flame structure at different ambient conditions
}

\author{
F. Payri ${ }^{a}$, J. M. García-Oliver ${ }^{a, *}$, R. Novella ${ }^{a}$, E. J. Pérez-Sánchez ${ }^{b}$ \\ a CMT - Motores Térmicos, Universitat Politècnica de València \\ Edificio 6D, Camino de Vera s/n, 46022, Valencia, Spain \\ Tel. (0034) 963877650 / Fax (0034) 963877659 \\ ${ }^{b}$ Barcelona Supercomputing Center - Centro Nacional de Supercomputación \\ Nexus II Building, C/ Jordi Girona 29, 08034, Barcelona, Spain \\ Tel. (0034) 934137716 / Fax (0034) 934137721
}

\begin{abstract}
Encouraged by the diversity of n-dodecane chemical mechanisms currently available, this investigation focuses on analysing the impact of using different fuel oxidation schemes on the diesel-like Engine Combustion Network (ECN) Spray A flame structure, simulated by means of an Unsteady Flamelet Progress Variable (UFPV) combustion model. The present research discusses systematically the characteristics of four n-dodecane chemical mechanisms in perfectly stirred reactors and counterflow laminar diffusion flames (flamelets) before the final evaluation in turbulent reacting sprays in order to describe the effects of adding different physical levels of complexity to the ignition of the mixtures. In addition, this analysis is complemented with the description of the effect of the boundary conditions on the flame structure.

Results evidence the extreme importance of the low temperature chemistry including the period for which the cool flame extends. The different prediction of this stage between mechanisms leads to noticeable different laminar flame structures which in turn produce substantially distinct turbulent flames, especially in the vicinity of the lift-off length (LOL) in terms of reactivity and
\end{abstract}

${ }^{*}$ Corresponding author. Email: jgarciao@mot.upv.es

Preprint submitted to Combustion and Flame

May 8, 2019 
positioning in the Z-T map. Finally, simulations confirm the strong effect of the boundary conditions, especially for the ambient temperature, on the ignitable mixtures which directly impacts on the soot precursors formation.

Keywords: Combustion modelling, Spray A, flamelet concept, Auto-ignition, Chemical mechanism

\section{Introduction}

During the last years the stringent pollutant emissions regulations have prompted research on efficient combustion strategies aimed at the minimization of such emissions. The use of exhaust-gas recirculation (EGR), high injection 5 pressures or more advanced concepts like Homogeneous Charge Compression Ignition (HCCI) [1] are only some few examples of the different attempts suggested in order to fulfil the regulation. Some of these strategies, like EGR, are widely used in commercial engines and, hence, their analysis and understanding is relevant.

10 However, obtaining local measurements of the spray in real engine conditions is a hard task and, therefore, spray modelling by means of Computational Fluid Dynamics (CFD) is still quite challenging. As a first step, it would be desirable to understand the spray in some ideal conditions to discard uncertainties and be able to apply the most advanced experimental techniques.

These concerns have promoted the definition by the Engine Combustion Network (ECN) [2] of a set of experiments that reproduce engine conditions to be performed in specific facilities by different institutions. Additionally, a considerable effort has been devoted to modelling in order to provide insight into the description of the combustion structure.

The well-known spray A from ECN is a single nozzle diesel spray where boundary conditions reproduce modern direct injection diesel engines conditions [2]. Characterization of spray A has been carried out in constant-volume preburn (CVP) combustion vessels and constant-pressure flow (CPF) rigs [3, 4. A $\mathrm{CPF}$ rig is available at CMT-Motores Térmicos whose experimental results are 
The spray is measured in terms of tip penetration, liquid length, ignition delay (ID) and lift-off length (LOL). Complementary information is extracted from optical measurements, such as Planar Laser-Induced Fluorescence (PLIF) or high-speed schlieren imaging [7].

30 of a rich partially premixed region, where soot is nucleated, and a diffusion flame, in the form of a thin reaction sheet at the location where stoichiometric mixture is found, which wraps up the spray, and in whose proximities, at the lean side, $\mathrm{NO}_{x}$ are produced. The first one is the chemical mechanism that describes the oxidation of the fuel. As hundreds of species are produced and consumed and thousands of reactions may take place during the fuel oxidation, it is almost unaffordable to deal with complete chemical schemes. Consequently, there is need of simplification that may lead to some loss of information.

More particularly, the complex alkanes autoignition process is known to be composed of a multi-stage combustion, with differentiated low and high temperature chemistries separated by a cool flame. Additionally, it is observed that, for homogeneous reactors, ignition delay curves typically show a negative 45 temperature coefficient (NTC) zone for temperatures around 900 and $1000 \mathrm{~K}$.

In consequence, low temperature chemistry is fundamental in order to predict ignition delays and plays a fundamental role in the chemical paths leading to the high temperature chemistry where the major species of combustion are produced 9, 10]. Additionally, pollutant emissions, and more particularly soot prediction, are a complex issue not solved yet. All these observations give a measure of the complexity involved in chemical modelling and the need of using detailed mechanisms that, however, can derive in a high computational cost. Notwithstanding, for modelling purposes, the combustion model is required to manage complex mechanisms in a feasible amount of time. 
describes how fluctuations in the turbulent flow and the chemical source terms are mutually affected in the frame of Reynolds Averaged Navier-Stokes (RANS) and Large Eddy Simulation (LES). This interaction is defined by the combustion model [11, 12].

It is evident that diesel ignition can only be solved in the frame of finite rate chemistry models. The most simple of them correspond to the well-stirred reactor (WSR) model where no turbulent fluctuations are retained. However, for diesel sprays the WSR model is not suitable, especially for low reactive conditions [13].

Models that account for TCI, such as Transported Probability Density Functions (TPDFs) methods and Conditional Moment Closure (CMC), have been applied to spray A simulation with very promising results [14, 15, 16, 17. However, both models imply a high computational cost making difficult its application to engine calculations.

The flamelet concept is another approach that has been applied to solve engine simulations as well as diesel sprays. The turbulent flame, which is assumed to develop in a thin layer when Damkhöler number is high, is described as an ensemble of strained laminar flames. TCI is accounted for by means of presumed probability density functions (PDFs) [18]. Combining this concept 75 with the Intrinsic Low Dimensional Manifold (ILDM), the Flame Prolongation of ILDM (FPI) and the Flamelet-Generated Manifold (FGM) approaches were formulated [19, 20]. Both methods define a manifold where the thermochemical paths are described by a reduced set of variables which are solved in the flow. Then, thermochemical information is retrieved from look-up tables depending on few inputs in order to solve transport equations. This turns out as a powerful concept since it decouples chemistry and TCI calculations from CFD and reduces the computational cost 21] allowing the use of complex chemical schemes.

Nonetheless, when this model is applied to engine calculations where conditions span over wide ranges and a great number of tables should be calculated, solving the flamelets requires a high computational cost when detailed chemistry 
is used. In this frame, the Approximated Diffusion Flamelet (ADF) model 22 . was suggested some years ago as an approach that reduces drastically such computational cost for the flamelet database generation. Satisfactory results have been reported with the use of this model [23, 24, 25] and it has been adopted for the calculations of this paper.

This brief review of the state of the art evidences that complex processes concurring in the spray preclude the use of simple mechanisms [26] and require combustion models that account for TCI [13].

This paper is focused on the analysis of spray A structure, representative of diesel sprays for modern engines, in quasi-steady and transient conditions. Special attention is deserved to the impact of the boundary conditions, in terms of ambient temperature and oxygen concentration, on the reactivity of the mixture and the spray structure. Additionally, the second objective of this paper is the evaluation of the influence of the chemical mechanism. The present contribution will show to which degree the differences observed among mechanisms under homogeneous condition persist in the spray calculation. While previous work has been carried out in this direction [27, 28, with special attention on the chemical aspects, the current analysis is mainly focused on the effect of the chemical mechanism on the laminar flames and the turbulent spray. Finally, a RANS turbulence model has been chosen due to the large number of simulations to be performed. This turbulence modelling approach has a clear interest for industrial applications and for this reason it is considered to be relevant within this particular research field [27, 29, 30, 31.

The paper opens with a description of the model as well as the numerical set-up and the boundary conditions for spray A. Subsequently, a preliminary evaluation of the chemical mechanisms for perfectly stirred reactors and, in a second step, for flamelet configurations is carried out. Spray A is then analysed in terms of flame global parameters such as ID and LOL. Then, the paper is divided in two main parts, the first one, being devoted to the auto-ignition process and, the second one, to the quasi-steady analysis. The conclusions of the work close the article. 


\section{Methodology}

\subsection{Description of the model}

This work is based on the approach developed in previous contributions by the authors for spray A [32, 33]. The model and the set-up are briefly described in the following.

An in-house CFD code was implemented in the open tool-box OpenFOAM environment 34 in the frame of RANS turbulence models. As cylindrical symmetry was imposed, a meridian plane, with dimensions 54 (radius) $\times 108$ (height) mm, was solved. The mesh was structured with a constant cell size of 0.25 (radial direction) $\times 0.5$ (axial direction) $\mathrm{mm}$ [35]. A standard $k-\varepsilon$ turbulence model was selected adjusting $C_{\varepsilon 1}=1.52$ in order to correct the spreading rate overestimation found for round jets [26].

The liquid phase was solved by means of a Lagrangian description in the frame of the Discrete Droplet Method (DDM) [36]. In this work, atomization and break-up were solved modelling the Kelvin-Helmholtz/Rayleigh-Taylor instabilities with constant values suggested in [35]. The number of parcels was chosen to inject a rate of $5.4 \cdot 10^{7}$ parcels $/ \mathrm{s}$ [35].

Regarding the combustion model, when facing diesel engine simulation, which requires the calculation of a great amount of boundary conditions, solving the complete flamelet model [12] for complex oxidation schemes becomes infeasible. This gave rise to the ADF approach where new hypotheses are assumed that lead to a simplified model which still maintains the physical structure of the

flamelet 22 . Since excellent results have been reported in the frame of diesel engines [22, 25, 37, 32, 38, the ADF model has been adopted for the current calculations.

The ADF model solves the transport equation 1 for the progress variable $Y_{c}$, which is defined as $Y_{c}=Y_{\mathrm{CO}}+Y_{\mathrm{CO}_{2}}$ [39, 22, 40].

$$
\frac{\partial Y_{c}}{\partial t}=\frac{\chi(a, Z)}{2} \frac{\partial^{2} Y_{c}}{\partial Z^{2}}+\dot{\omega}_{c}^{H R}\left(Z, Y_{c}\right)
$$


The chemical source term for the progress variable $\dot{\omega}_{c}^{H R}$ is defined as the chemical source term for this variable, given by a set of homogeneous reactors (HRs) that auto-ignite with the same initial and boundary conditions that the flamelet and evolve at constant pressure with no heat loses. Such evolution is gathered in a database with $\left(Z, Y_{c}\right)$ as input variables and its information is retrieved when solving equation 1 . Once equation 1 is solved, the temporal evolution for the reactive scalars during flamelet ignition is retrieved by means of interpolations from the homogeneous reactors database and the profile $Y_{c}(t)$ obtained for each mixture fraction and strain rate.

The scalar dissipation rate, $\chi$, which measures the strength of convection and diffusion, is defined by $\chi=2 D|\nabla Z|^{2}$ and is approximated by the profile [1]

$$
\chi(a, Z)=\frac{a}{\pi} Z_{s}^{2} \exp \left[-2\left(\operatorname{erfc}^{-1}\left(2 Z / Z_{s}\right)\right)^{2}\right]
$$

where $a$ the strain rate (assumed to be constant in the whole flame) and $Z_{s}$ the saturation mixture fraction, which is the maximum mixture fraction for which the fuel does not condensate [41. Notice that $Z_{s}$ is the upper limit for the mixture fraction since $Z$ only accounts for the gaseous phase.

In addition, to define the chemical database, the progress variable is normalized according to

$$
c=\frac{Y_{c}-Y_{c}^{\text {inert }}}{Y_{c}^{\text {equil }}-Y_{c}^{\text {inert }}}
$$

where $Y_{c}^{\text {inert }}$ and $Y_{c}^{\text {equil }}$ are the inert and equilibrium values for the progress variable. By virtue of the monotonic evolution of $Y_{c}$ with time, $c$ ranges between 165 0 and 1.

To reduce numerical uncertainties, a fine discretization has been chosen in the different directions. For the mixture fraction approximately 160 values, clustered around the stoichiometric value, were taken while for the progress variable direction 504 points were retained and around 40 strain rates were 

generate the flamelet solutions is described in detail in 38 .

TCI is accounted for by means of presumed PDFs for mixture fraction and scalar dissipation rate assuming statistical independence between both variables [11. A beta-PDF, used to model mixture fraction fluctuations, is defined by 175 the average value, $\widetilde{Z}$, and its variance $\widetilde{Z^{\prime \prime 2}}$. For the scalar dissipation rate a lognormal is assumed where $\sigma^{2}$, related to $\widetilde{\chi}_{s t}$ variance, is fixed constant and equal to 2 [29]. The re-parametrization of the solutions in terms of $\widetilde{Y}_{c}$ rather than time leads to a turbulent combustion manifold in the form $\widetilde{\psi}=\widetilde{\psi}\left(\widetilde{Z}, \widetilde{Z^{\prime \prime 2}}, \widetilde{\chi}_{s t}, \widetilde{Y}_{c}\right)$.

In this work and depending on the boundary conditions, around 32 values 180 have been tabulated for $\widetilde{Z}, 17$ for $\widetilde{Z^{\prime \prime 2}}$ and 35 for $\widetilde{\chi}_{s t}$ while 51 for $\widetilde{Y}_{c}$ with a parabolic distribution in order to retain accurately auto-ignition.

Assuming a proportionality between convective and mixing turbulent times, $\tilde{\chi}$ is modelled as 12

$$
\widetilde{\chi}=C_{\chi} \frac{\varepsilon}{k} \widetilde{Z^{\prime \prime 2}}
$$

where $C_{\chi}$ is a constant and $k$ and $\varepsilon$ are the turbulent kinetic energy and its dissipation rate.

The chemical source term required to solve CFD transport equations is found from the difference between the next value predicted by the turbulent manifold and the current value in the cell divided by the time step. More details can be found in $42,32,33,43$.

Finally, four well-known mechanisms for n-dodecane have been used for these calculations to elucidate their influence on the reactive spray solution. Starting from the simplest one, the chosen mechanisms are Yao et al. [10, a skeletal mechanism with 54 species and 269 reactions, Wang et al. 44] which describes n-dodecane oxidation with 100 species and 432 reactions, Narayanaswamy et al. [9, which is an intermediate mechanism including 255 species and 2289 reactions, and, finally, Cai et al. scheme [45], which includes 1692 species and 11015 reactions. Due to the large number of participating species and reactions, the 
latter mechanism can only be used with combustion modelling approaches such as the one presented here. These mechanisms have been thoroughly analysed in homogeneous conditions comparing experimental measurements in a rapid compression-expansion machine and modelling results [28. The latter study is complemented with the current work, where special attention is devoted to the chemical scheme effect on laminar flames as well as the turbulent reacting spray.

\subsection{Parametric study}

The boundary conditions of the parametric study for spray A correspond to those experimentally measured at CMT-Motores Térmicos in the CPF facility ([5, 6, 46]). This facility is a CPF rig where the ambient gas thermochemical conditions are controlled by supplying an oxygen and nitrogen mixture compressed and pre-heated before entering into the open combustion chamber in which the fuel is injected. For spray A fuel is dodecane, which is a diesel surrogate fuel.

The nominal case is defined by the ambient temperature, oxygen concentration and density given by $T_{a m b}=900 \mathrm{~K}, X_{O_{2}}=0.15$ and $\rho=22.8 \mathrm{~kg} / \mathrm{m}^{3}$ and three parametric sweeps are carried out. The first two span the temperature from $750 \mathrm{~K}$ to $900 \mathrm{~K}$ with $X_{O_{2}}=0.15$ and 0.21 . To simulate the EGR effect a third parametric variation in the oxygen concentration direction is performed with $0.13 \leq X_{O_{2}} \leq 0.21$ at the nominal ambient temperature. All the cases keep density at the nominal value.

For all the studies the injection pressure is fixed at $150 \mathrm{MPa}$ and a long injection rate ( $>4 \mathrm{~ms}$ ), obtained from the virtual injection rate generator [46], is imposed in order to reach a quasi-steady state. Nominal injector diameter is $90 \mu \mathrm{m}$, with nozzle code 210675 [2] and discharge coefficient equal to 0.9 [47]. The fuel temperature is assumed constant at $363 \mathrm{~K}$.

The boundary conditions are gathered in table 1 where $Z_{s t}$ refers to the stoichiometric mixture fraction. 
Table 1: Definition of the spray A parametric studies.

\begin{tabular}{ccccc}
\hline$X_{O 2}$ & $T_{a m b}(\mathrm{~K})$ & $\rho_{a m b}\left(\mathrm{~kg} / \mathrm{m}^{3}\right)$ & $p_{i n j}(\mathrm{MPa})$ & $Z_{s t}$ \\
\hline \hline 0.13 & 900 & 22.8 & 150 & 0.040 \\
\hline 0.15 & 750 & 22.8 & 150 & 0.046 \\
\hline 0.15 & 800 & 22.8 & 150 & 0.046 \\
\hline 0.15 & 850 & 22.8 & 150 & 0.046 \\
\hline 0.15 & 900 & 22.8 & 150 & 0.046 \\
\hline 0.21 & 750 & 22.8 & 150 & 0.063 \\
\hline 0.21 & 800 & 22.8 & 150 & 0.063 \\
\hline 0.21 & 900 & 22.8 & 150 & 0.063 \\
\hline
\end{tabular}

225

In order to establish a clear sequence of how combustion proceeds when different processes interact among them, this section opens with the analysis of combustion in well-stirred conditions, after which results are compared to the combustion evolution when it is affected by diffusion in laminar conditions, corresponding to the flamelet calculations. Subsequently, the section follows with the description of the turbulent combustion in CFD computations. This part first analyses the global reactive parameters in order to validate the model. Then the spray structure is described during auto-ignition and quasi-steady state in order to figure out the effect of the boundary conditions as well as the influence of the chemical mechanism.

\subsection{Homogeneous reactors and flamelet results}

Previous to the analysis of spray A conditions, a first assessing of the mechanisms is shown in figure 1 where the ignition delay for homogeneous reactors auto-ignition is plotted as a function of the inverse of the temperature for the four analysed mechanisms and the shock tube experimental data from 48. The figures show the behaviour for equivalence ratio $\phi=0.5$ and 1 with an initial pressure $p=20 \mathrm{~atm}$. Calculations are performed imposing constant volume and adiabatic evolution. The ignition delay for the simulations is defined in a similar way as in the experiment [48, that is, the time when the temporal derivative of

From experiments a soft NTC region in the temperature interval $[800,900]$ $\mathrm{K}$ is observed that seems to be stronger for the stoichiometric mixture. The 

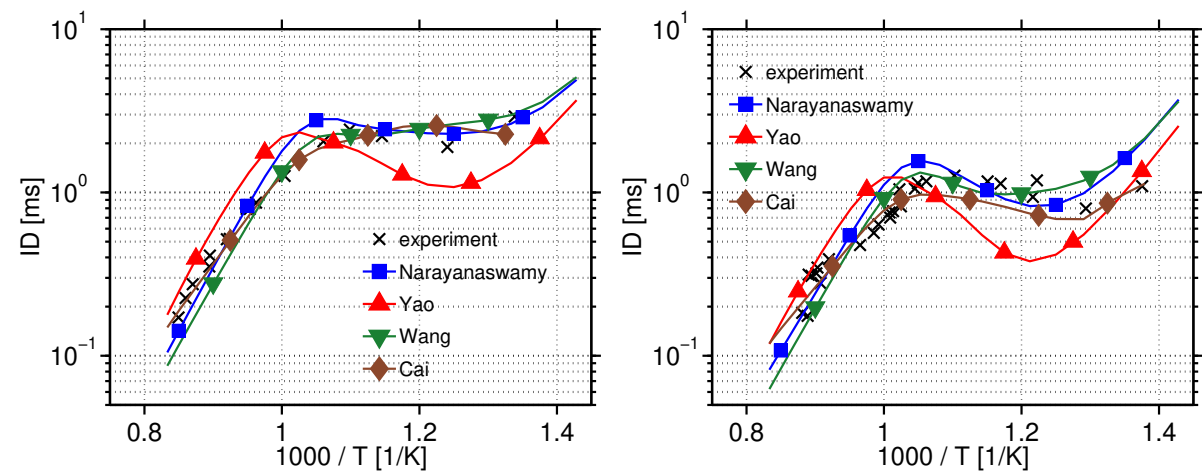

Figure 1: Experimental and modelled ignition delays as a function of the temperature for $\phi=0.5$ (left) and 1 (right). Calculations for pressure $p=20$ atm.

Yao mechanism is the one that shows more prominent differences compared to the experiment and the other mechanisms, especially in the NTC region, with a sharper decrease in ignition delay. On the contrary, Narayanaswmay and, especially, Wang and Cai results are very similar in the whole range of temperatures and provide ignition delays remarkably close to the experimental ones. Wang scheme together with Cai describe a weak NTC region and in the case of the lean mixture for Wang this region is not present. Finally, Yao provides noticeable faster ignition delays than the other mechanisms for temperatures $<$ $1000 \mathrm{~K}$ while it is slower in the high-temperature zone (temperatures $>1000$ $\mathrm{K})$.

From this initial assessment, Yao is expected to evolve faster than the other mechanisms in subsequent calculations, since the simulated spray A conditions correspond to temperatures lower than $1000 \mathrm{~K}$. However, the previously observed mechanism ordering is not always preserved, emphasizing the importance of the first ignition stage, which is not described in figure 1 since the sharp increase of pressure that defines the ignition delay is detected during the second ignition stage.

After this preliminary evaluation for a fixed composition and initial temperature, figure 2 shows the ignition delays for spray A nominal condition for low and high temperature stages, which are defined as the time spent to reach the 
initial temperature plus 30 and $400 \mathrm{~K}$, respectively, and are referred to as $\mathrm{ID}_{1}$ and $\mathrm{ID}_{2}$, respectively. These Ignition delays are represented as a function of $Z$ for the four mechanisms. Note that the cool flame period elapses between $\mathrm{ID}_{1}$ and $\mathrm{ID}_{2}$.

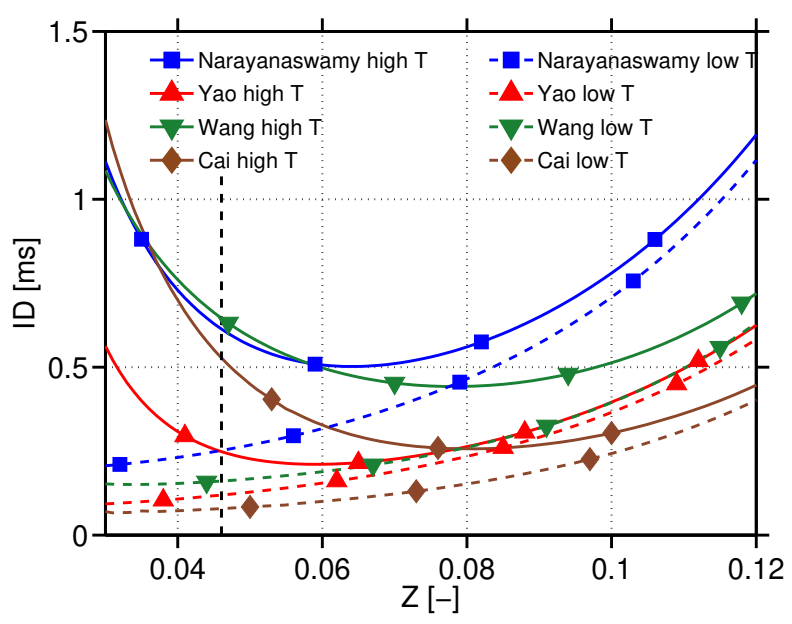

Figure 2: Ignition delay for a set of reactors starting from the adiabatic mixing curve derived for the spray A nominal condition. Both the low and high temperature stages have been quantified for the four simulated mechanisms Vertical black dashed line indicates the stoichiometric mixture fraction.

From figure $2 \mathrm{ID}_{1}$ is observed to increase with the mixture fraction as well as its slope. Cai mechanism is the fastest, followed by Yao and Wang which show very similar ignition delays for this stage since they move parallel. 275 Narayanaswamy scheme predicts the most dilated first ignition delay. This is a first evidence that the initial mechanism ordering observed in figure 1 is not held in low temperature stages.

Moreover, it is observed that the cool flame period $\left(\mathrm{ID}_{2}-\mathrm{ID}_{1}\right)$ becomes shorter with mixture fraction increase, being almost non-existent for Yao and moderate for Narayanaswamy and Cai while Wang mechanism models the longest one. This shortening of the cool flame interval when enriching the mixture favours the displacement of combustion to rich mixtures and freezes temporally the ignition for lean mixtures as will be discussed.

Regarding $\mathrm{ID}_{2}$ it is seen that, different from $\mathrm{ID}_{1}$, it has a minimum for the 
285 and Narayanaswamy, although is slightly lower for Yao $\left(Z_{M R}=0.059\right.$ for Yao while it values 0.063 for Narayanaswamy), while for Wang and Cai this value is displaced to moderately rich mixtures $\left(Z_{M R}=0.077\right.$ for Wang and 0.08 for Cai). Yao mechanism is the one which shows the shortest $\mathrm{ID}_{2}$ for $Z<0.8$ while Narayanaswamy and Wang schemes predict very similar $\mathrm{ID}_{2}$ as shown in figure 1. However, the behaviour for rich mixtures is drastically different, as figure 2 reveals, since Narayanaswamy scheme predicts very long $\mathrm{ID}_{1}$ that take even a longer time than $\mathrm{ID}_{2}$ for Wang mechanism. In addition, the fact that Yao produces the shortest $\mathrm{ID}_{2}$ for the lean range, where all other three mechanisms collapse is another remarkable result.

One of the most important conclusions is that all the mechanisms predict that the low temperature ignition starts at lean mixtures $\left(\mathrm{ID}_{1}\right.$ is lower for lean mixtures) due to their higher initial temperature. However, the reduction or even the disappearance of the cool flame period when moving towards richer mixtures results a faster high temperature ignition for the rich mixtures than for the lean ones. This has been one of the arguments used to explain the displacement of combustion from lean mixtures, where the first ignition kernels appear in spray A, to rich mixtures where the second stage and high temperature ignition is observed [50, 51]. Finally, it is worth mentioning that the behaviour described for the ignition delay in figure 2 is qualitatively observed at other boundary conditions.

In order to deepen in the ignition process for the different mechanisms, the ignition delay information is complemented with the map of the chemical source terms for the progress variable from the homogeneous reactors, $\dot{\omega}_{c}^{H R}\left(Z, Y_{c}\right)$, for the nominal case. This is shown in figure 3 where, in order to better visualise the whole map, a logarithmic scale has been used. 

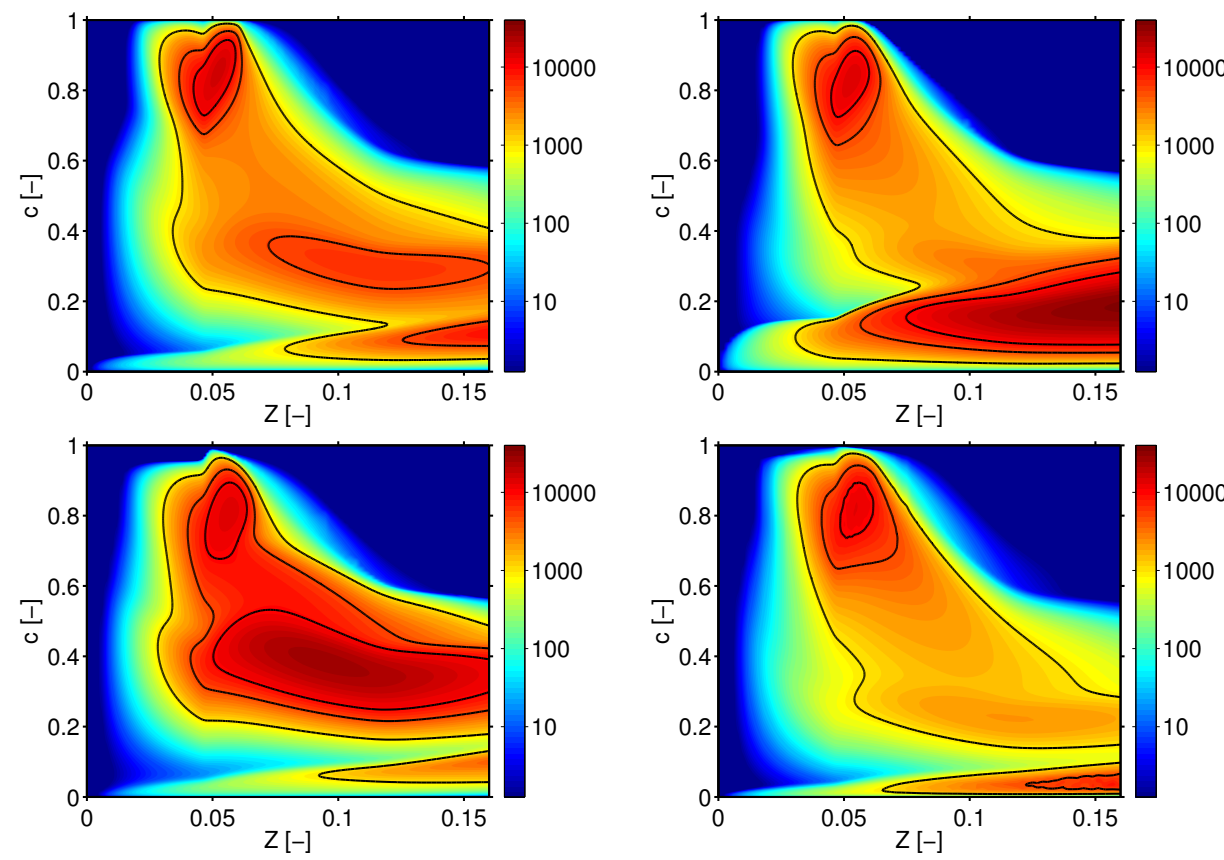

Figure 3: Chemical source terms for the progress variable for homogeneous reactors with nominal condition. Top: Narayanaswamy (left), Yao (right), bottom: Wang (left) and Cai (right) mechanisms. $\dot{\omega}_{c}^{H R}$ level curves for values 1000,5000 and $100001 / \mathrm{s}$ are represented for reference. Scales are common for all figures.

In figure 3 different regions of intense chemical activity are identified depending on the chemical mechanism. All of them predict high chemical source terms for low $c$ values and rich mixtures that increase when augmenting $Z$. This zone corresponds to the low temperature ignition and is followed by an important reduction of the chemical source term during auto-ignition that is related to the cool flame period (time elapsed between $\mathrm{ID}_{1}$ and $\mathrm{ID}_{2}$ ). Clearly, such period does not extend on a wide interval of the progress variable (as well as temperature) since the low chemical activity only produces a modest increase of these variables. The cool flame is followed by an extremely high increase of the source term that corresponds to the high temperature stage which is specially 
intense for stoichiometric and slightly rich mixtures.

Although a similar qualitative behaviour is observed among different mechtivity freezes and diffusion is the only mechanism to propagate ignition. On the contrary, having a relatively high chemical source term during the cool flame, as in the case of Yao, promotes the continuation of combustion by chemical reaction together with diffusion transport.

In order to clarify species and temperature dependence with intrinsic combustion variables during homogeneous reactors auto-ignition, their temporal evolutions are drawn as a function of $c$ for several mixtures. In figure 4 the evolution of formaldehyde $\left(\mathrm{CH}_{2} \mathrm{O}\right)$, hydrogen peroxide $\left(\mathrm{H}_{2} \mathrm{O}_{2}\right)$, carbon monoxide 
and carbon dioxide ( $\mathrm{CO}$ and $\mathrm{CO}_{2}$, respectively) mass fractions (denoted by $Y$

on the axis) are shown, together with the temperature. In order to share the same axis they are multiplied by a factor. For reference, the $c$ values corresponding to an increase of the initial temperature of 30 and $400 \mathrm{~K}$ (see $\mathrm{ID}_{1}$ and $\mathrm{ID}_{2}$ definitions) are included.
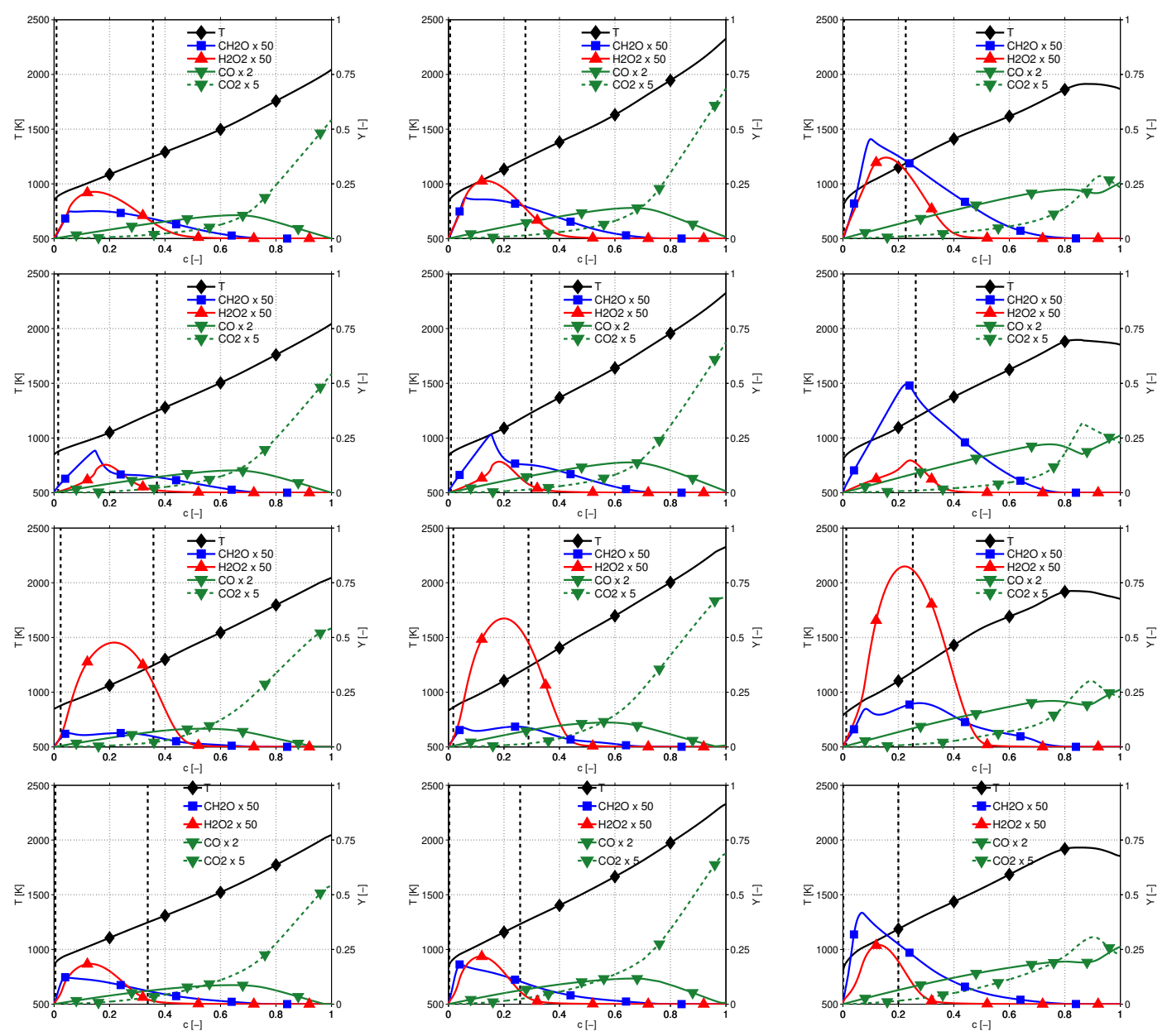

Figure 4: Temperature and species mass fractions evolution as a function of $c$ for Narayanaswamy (top row), Yao (second row), Wang (third row) and Cai (last row) for $Z=0.035$ (left column), 0.046 (center column), 0.08 (right column). Vertical lines correspond to the $c$ values for the low and high temperature ignition delay criteria from figure 2 Results for nominal conditions. 
It is observed that temperature and intermediate and final products $\mathrm{CO}$ simulations.

Finally, as the turbulent flame is considered to be composed of an ensemble of embedded laminar flames, analysing the flamelet behaviour for the different 
mechanisms is the next natural step, for which diffusion may become as intense as the chemical reactions. The structure of combustion is represented in figure 5 where the transient evolution and the steady solutions of the flamelets are described by several $Z-T$ profiles for different strain rates, namely, 10, 100 and $1000 \mathrm{1} / \mathrm{s}$, which cover the auto-ignition range. This auto-ignition range is defined as the interval of strain rates in which the flamelet can evolve from the inert conditions to the high temperature steady solution of the S-curve 12. In figure 5, profiles corresponding to the first ignition as well as curves for which the second ignition is developed for the fastest mixtures have been represented. 

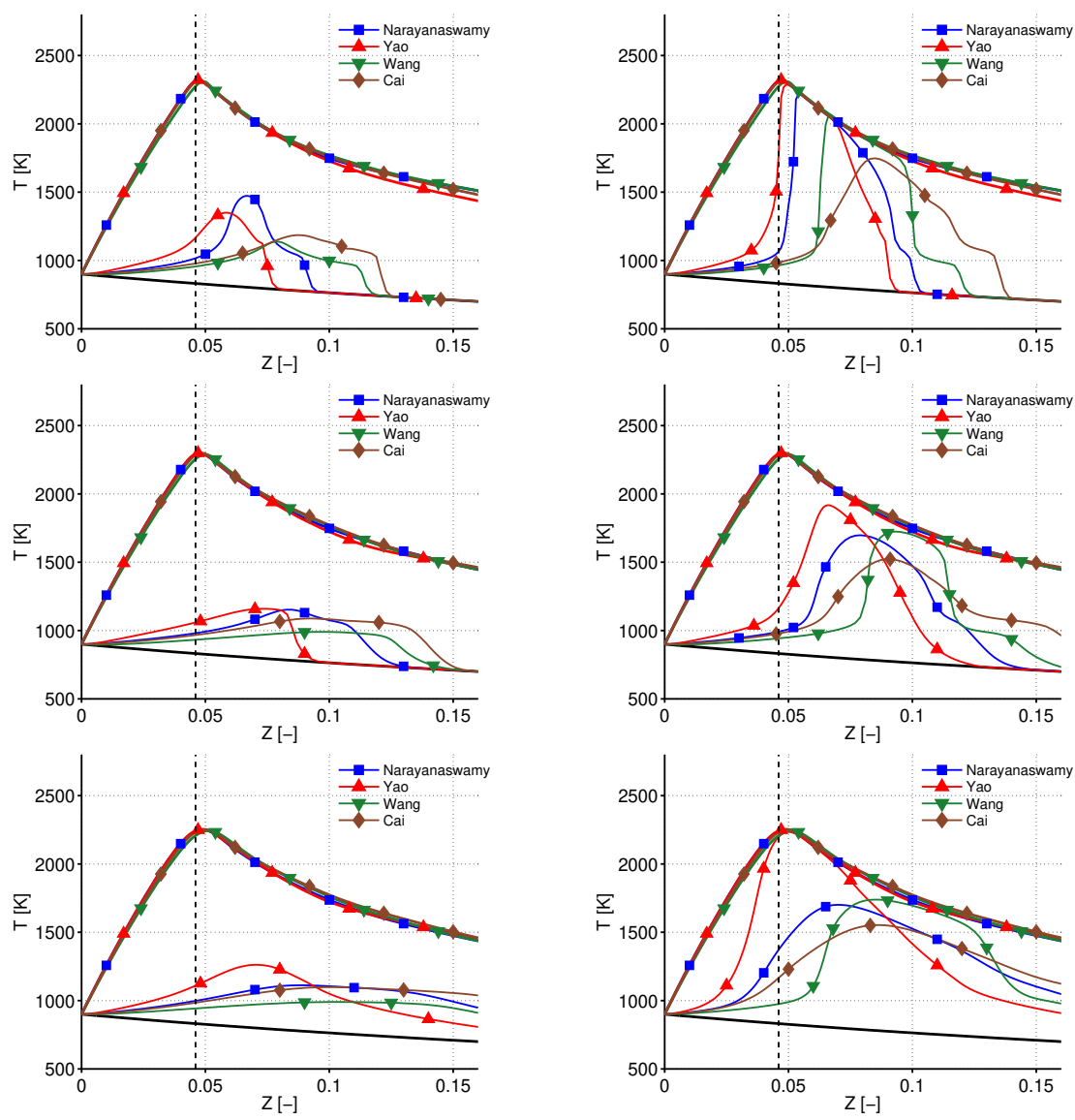

Figure 5: Structure of the laminar combustion in terms of $Z-T$ for the set of mechanisms for nominal condition. The figures correspond to strain rates $101 / \mathrm{s}$ (top row), $1001 / \mathrm{s}$ (second row) and $1000 \mathrm{1} / \mathrm{s}$ (bottom row). Left column: profiles corresponding to first ignition. Right column: profiles corresponding to second ignition. Stoichiometric mixture is indicated with a black dashed line.

In all the cases ignition starts at lean mixtures, which corresponds to the first ignition stage, and there is a subsequent displacement to richer mixtures [17. This is in agreement with figure 2 where it is observed that the shortest $\mathrm{ID}_{1}$ is found for the lean mixtures as a consequence of their higher initial temperature [50, 51]. Once the first ignition for low temperature has proceeded, the cool flame period starts. Figure 2 shows how lean mixtures, in spite of their short $\mathrm{ID}_{1}$, have the longest cool flame period since this interval is reduced when enriching 
the mixture. In agreement with this effect, figure 5 shows how lean and slightly rich mixtures remain frozen while the second ignition occurs in richer mixtures.

When $Z_{M R}$ overtakes its cool flame period it initiates the high temperature stage that leads to the steady solution. The different states in the ignition process between mixtures that remain in the cool flame and those that are close to the steady solution generate a strong gradient, that depending on the strain rate may be localized or, on the contrary, extend over a wide region of mixtures. Diffusion generates a flux of energy and radicals that tends to propagate ignition towards adjacent mixtures.

15 The different morphology of combustion for the investigated mechanisms is due to the different values for $Z_{M R}$ as well as the latency period related to the cool flame and, ultimately, is due to the different chemical source terms. In the case of Yao mechanism, $Z_{M R}$ is positioned in the slightly rich mixtures $(0.06$ approximately although there is a mild dependence of $Z_{M R}$ with the strain rate) and the cool flame period is extremely short for $Z>Z_{M R}$. Consequently, in the case of low strain rates, the first stage combustion only has developed until $Z=0.07$, approximately, when the second ignition starts in the vicinity of $Z_{M R}$. This means that a very reduced range of rich mixtures have initiated their first ignition when $Z_{M R}$ commences the second ignition stage. This second ignition is extremely fast and generates strong gradients that diffuse the progress variable to the surrounding mixtures. On the contrary, for the Wang mechanism $Z_{M R}$ is found at very rich mixtures (around 0.08) and there exists a long cool flame period. This makes it possible for a wide range of rich mixtures to evolve and reach the cool flame before the vicinity of $Z_{M R}$ starts the second ignition, as observed in figure 5. In the case of Cai scheme, as observed from figure 2, even if the latency period between the different combustion stages is not so prolongated as in Wang, combustion is very displaced to rich mixtures $\left(Z_{M R}\right.$ around 0.08$)$ as figure 5 confirms.

In addition, the profile of the curves can be described with the help of the chemical source terms from figure 3 . In figure 5 it is observed how the layer that separates the mixtures close to the steady solution from those found in the cool 
flame period is thin for the Wang (sharp profile observed in this layer) while for the Yao extends in a wide region (smoother profile observed in this layer). In the case of Wang mechanism, this is explained by the extremely low chemical source terms found during the cool flame period (between first and second stage ignitions) which produce so long latency intervals during this period as deduced from figure 2 Hence, chemical activity is extremely low once the first ignition is finished and, in consequence, diffusion is the only mechanism that propagates combustion in this layer. Once enough progress variable is transported to the surrounding mixtures, the chemical activity becomes extremely intense and these mixtures evolve rapidly to the steady state generating the sharp profile in the interface between mixtures close to the steady value and mixtures in the cool flame period. Clearly, increasing the strain rate mitigates this behaviour and tends to transport the progress variable to further mixtures smoothing the profiles.

On the contrary, when analysing the chemical source terms for Yao it was pointed that it predicts moderate values during the cool flame period. Then combustion advances not only as a consequence of the diffusion transport but due to the moderate chemical activity too, which reduces the gradients between mixtures and leads to a softer profile in the region that separates the high and low temperature chemistry compared to Wang solutions. Again Narayanaswamy shows an intermediate behaviour between those described for Yao and Wang while Cai predicts smooth profiles at very rich mixtures as a consequence of the low/moderate chemical source terms after the cool flame (see figure 3).

Finally, the increase of strain rate, and consequently of diffusion fluxes, reduces the progression of the most reactive mixtures during the second stage since an increasing fraction of their production is distributed to the surrounding mixtures. This results in richer mixtures reaching the cool flame period before the second ignition eventually develops, which is evidenced by the spreading of combustion over wider mixture regions (see figure 5).

Similar figures to 5 are shown in figures 6 and 7 in order to analyse the effect of the boundary conditions. 

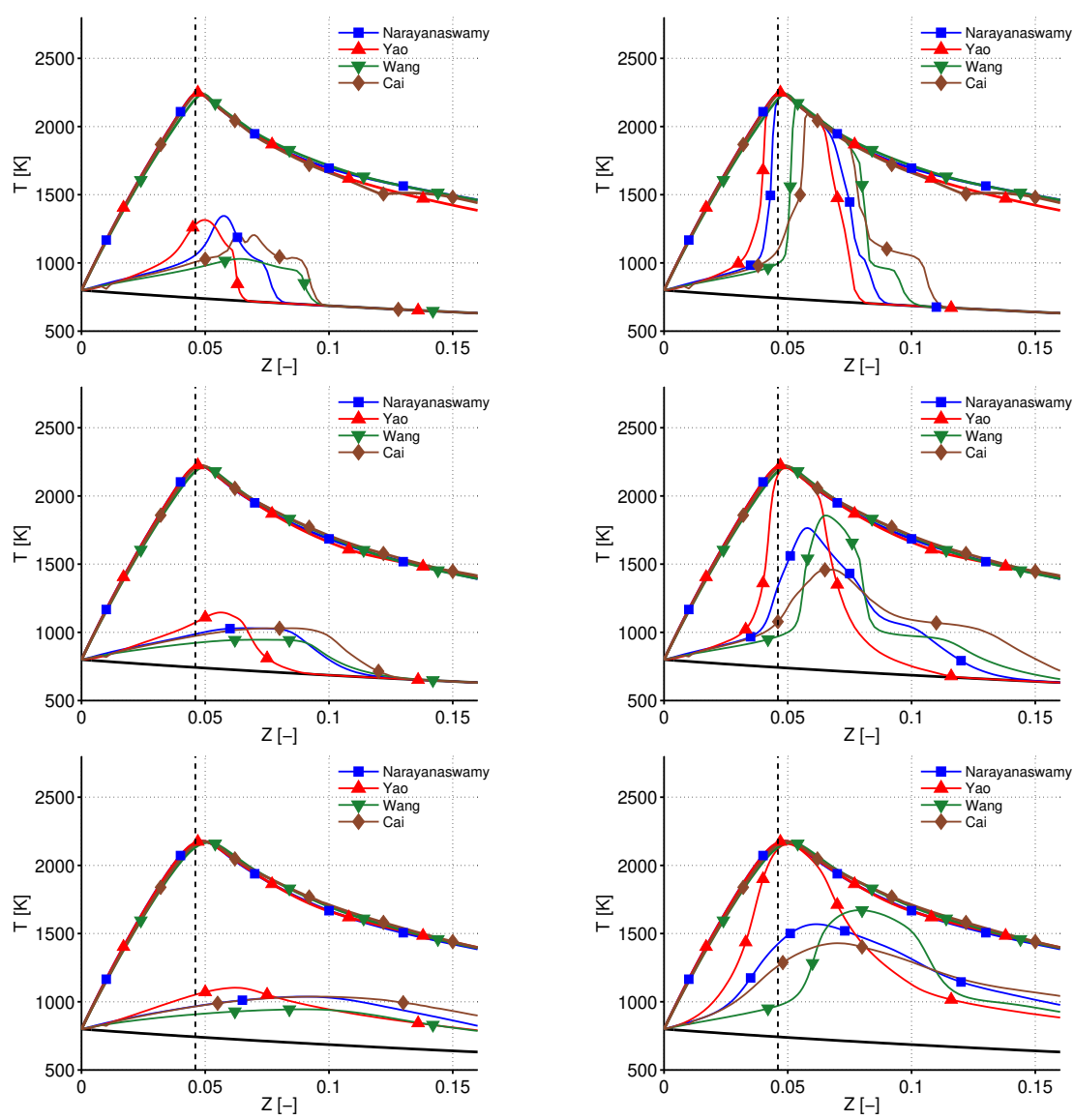

Figure 6: Structure of the laminar combustion in terms of $Z-T$ for the set of mechanisms for $T_{a m b}=800 \mathrm{~K}$ and $X_{\mathrm{O}_{2}}=0.15$. The figures correspond to strain rates $101 / \mathrm{s}$ (top row), 100 $1 / \mathrm{s}$ (second row) and $10001 / \mathrm{s}$ (bottom row). Left column: profiles corresponding to first ignition. Right column: profiles corresponding to second ignition. Stoichiometric mixture is indicated with a black dashed line. 

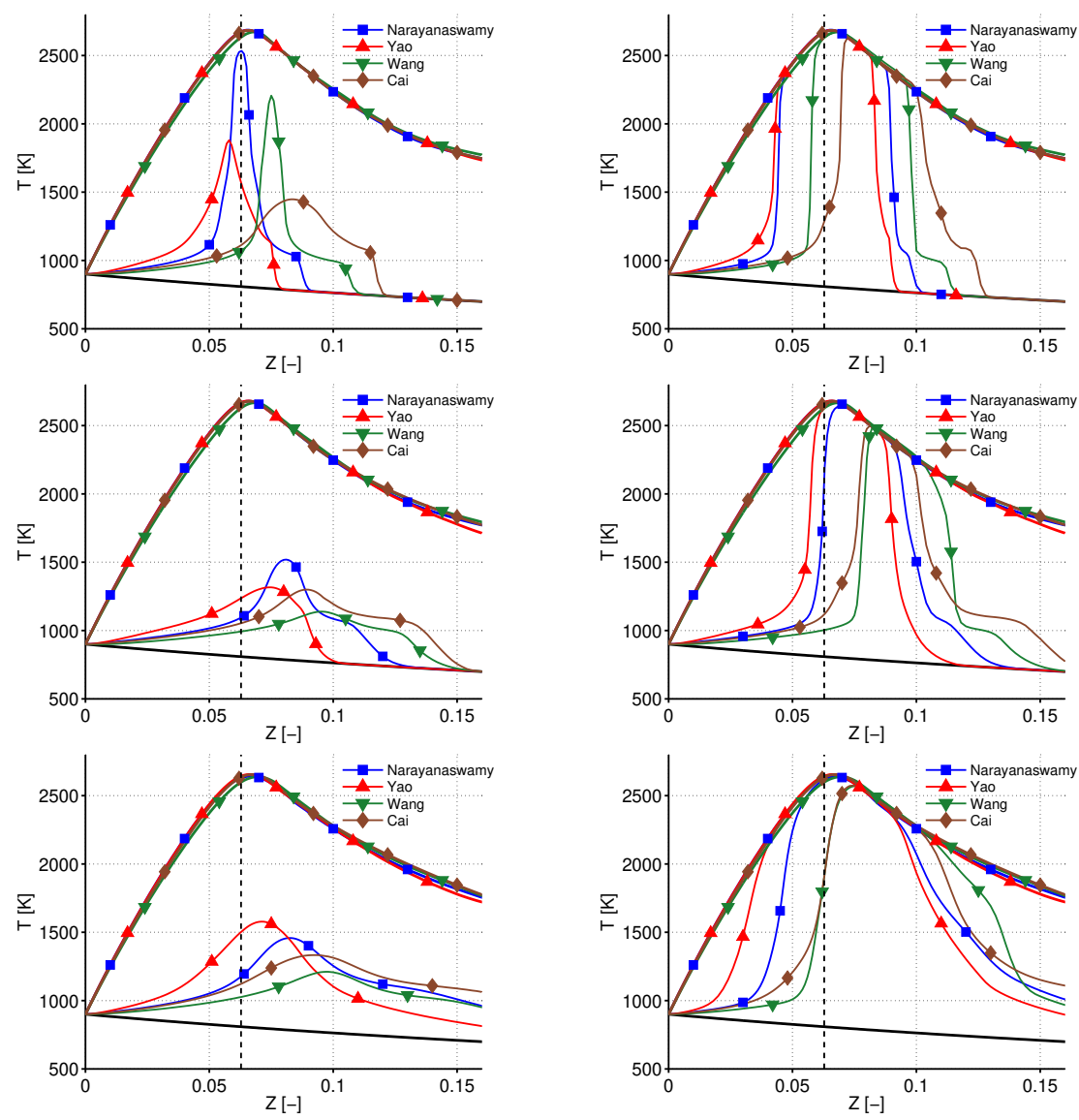

Figure 7: Structure of the laminar combustion in terms of $Z-T$ for the set of mechanisms for $T_{a m b}=900 \mathrm{~K}$ and $X_{\mathrm{O}_{2}}=0.21$. The figures correspond to strain rates $101 / \mathrm{s}$ (top row), 100 $1 / \mathrm{s}$ (second row) and $10001 / \mathrm{s}$ (bottom row). Left column: profiles corresponding to first ignition. Right column: profiles corresponding to second ignition. Stoichiometric mixture is indicated with a black dashed line.

Regarding the ignition behaviour for each mechanism, similar conclusions than those drawn for the nominal case are applied to the other boundary conditions. Comparing among different boundary conditions, it is observed that reducing the ambient temperature displaces the whole combustion and, in particular $Z_{M R}$, to leaner mixtures implying a reduction of reactivity of rich mixtures.

The oxygen concentration increase provokes that the most reactive mixtures 
augment strongly their chemical source term sharpening the slope of the region that separates the mixtures found in their second stage ignition and the mixtures which remain in the cool flame period. Additionally, according to figures 5 and 7. $Z_{M R}$ does not depend on the oxygen concentration.

Finally, it is worth mentioning that higher levels of diffusion may be supported by the flame in the auto-ignition range when increasing the ambient temperature and the oxygen concentration as a consequence of the higher mixture reactivity.

It stems from this detailed analysis that the low temperature chemistry, and more particularly the period for which the cool flame extends as well as the relative duration of this period between different mixtures, is very relevant in determining the evolution of the laminar flame and may lead to substantially distinct combustion structures as observed for the different chemical mechanisms. A second conclusion is, as previously explained, that the selection of the chemical scheme has a large impact on the radicals production especially during the low temperature stage, which is the most affected by the choice of the oxidation mechanism, in contrast with the high temperature regime where all of them show a clear agreement.

\subsection{Analysis of the reactive spray global parameters}

Previous work developed by the authors [32] showed that an excellent agreement is achieved with the chosen configuration of the model for the tip and liquid penetrations of the spray as well as the mean mixture fraction and its variance in inert conditions supporting further analysis in reacting conditions. This part will be omitted here for brevity reasons. The description of the reacting spray evolution starts with the investigation of global parameters, namely, the ignition delay and the lift-off length, in order to assess the capabilities of the model and sustain subsequent analysis.

Ignition delay (ID) and lift-off length (LOL) are experimentally both determined by chemiluminescence, ID as the time spent to reach $50 \%$ of the maximum high-temperature chemiluminescence level and LOL as the minimum axial loca- 
tion where $50 \%$ of the leveling-off value of $\mathrm{OH}^{*}$ chemiluminescence is observed [2]. For modelling purposes different definitions have been suggested in the literature. In the frame of the ECN, ID is calculated as the time spent from start of injection (SOI) until the maximum rise of maximum Favre-averaged temperature takes place. LOL is defined as the minimum axial distance to the nozzle where $14 \%$ of the maximum value of $\widetilde{Y}_{\mathrm{OH}}$ in the domain is reached [2]. However, in this work, it has been observed that the ECN ignition delay criterion may provide ignition delays associated with high temperatures close to $2000 \mathrm{~K}$. Additionally, a criterion based on a temporal derivative is prone to be affected by small perturbations in the temperature profile that may distort the measurement specially for slow ignitions. Hence, a criterion defined as the time spent from SOI until the maximum Favre-averaged temperature reaches the ambient temperature plus $400 \mathrm{~K}$ is adopted in this work [26]. A value of $400 \mathrm{~K}$ discards measuring ignition delay during cool flames combustion nor it is too high to be biased towards the last steps of the transient ignition. Regarding LOL, ECN criterion is adopted.

Figures 8, 9 and 10 gather results related to ID and LOL for all the analysed parametric sweeps where the experimental measurements correspond to those measured at CMT-Motores Térmicos [5].
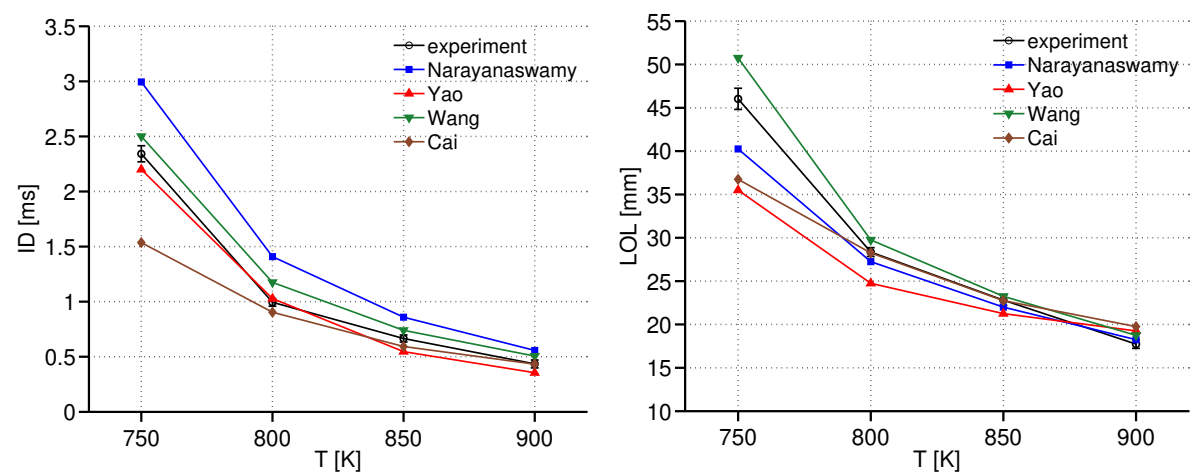

Figure 8: ID (left) and LOL (right) for the temperature parametric variation with $X_{\mathrm{O}_{2}}=0.15$. In both figures error bars indicate the experimental uncertainty.

These figures show a very good agreement between the modelled results 

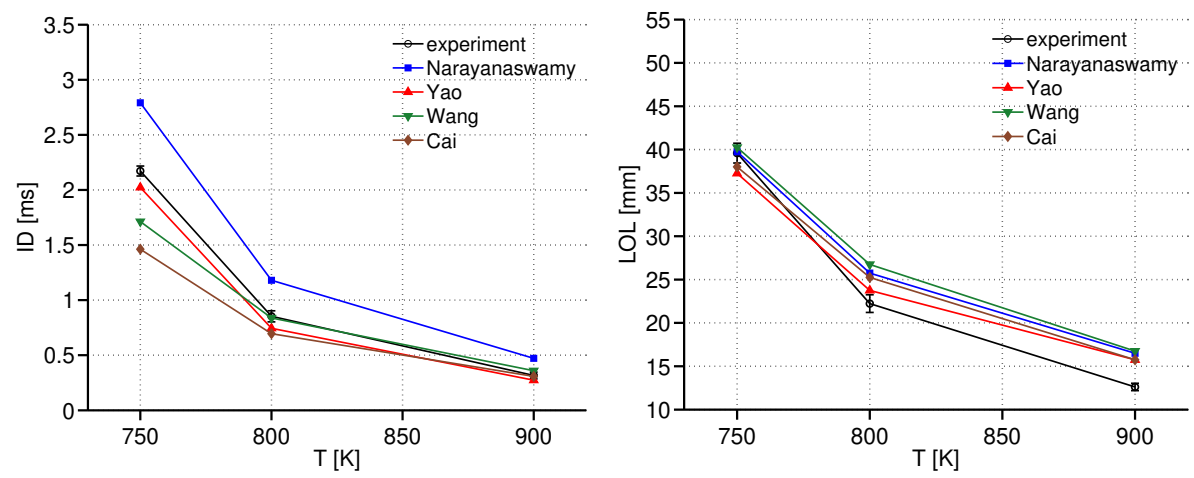

Figure 9: ID (left) and LOL (right) for the temperature parametric variation with $X_{\mathrm{O}_{2}}=0.21$. In both figures error bars indicate the experimental uncertainty.
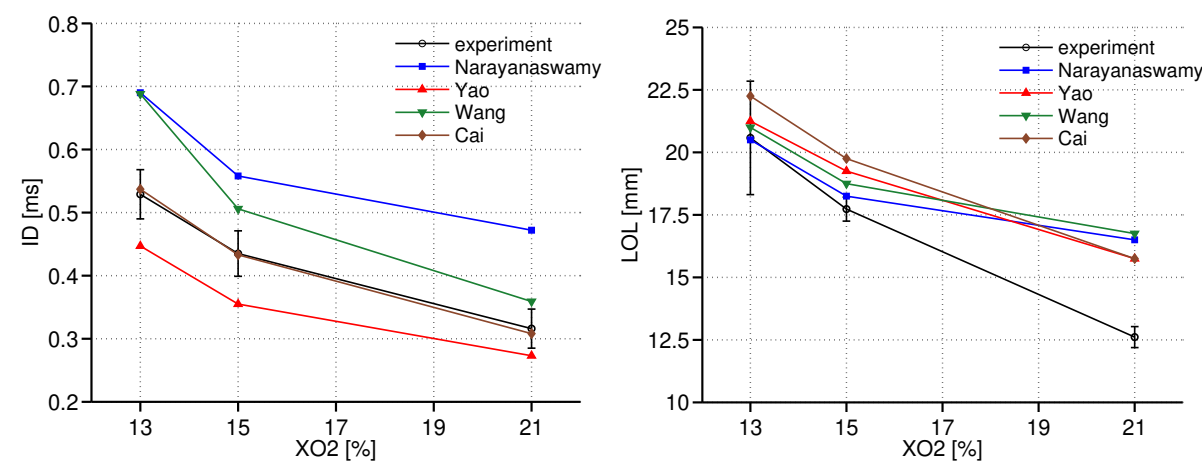

Figure 10: ID (left) and LOL (right) for the oxygen parametric variation with $T_{a m b}=900 K$. In both figures error bars indicate the experimental uncertainty.

and the experiments and, hence, demonstrate that the ADF model is able to reproduce diesel combustion with similar results to those found in the literature [53. In general, Narayanaswamy scheme provides the longest ignition delays, which, in addition, are slightly longer than experiments, followed by Wang while Yao and Cai mechanisms are the ones that predict the shortest ignition delays. This ordering was indicated in figures 1 and 2 for homogeneous reactors for Narayanaswamy, Yao and Wang schemes and, in consequence, figures 8, 9 and 10 show that it is preserved at all the stages of auto-ignition for such schemes. Cai mechanism predicts excellent ignition delays, with remarkable results for the oxygen sweep, although shows some deviations for very low temperatures. 
It is interesting to note that the aforementioned ordering for ignition delays is not observed for lift-off lengths (in fact, Narayanaswamy being the slowest mechanism provides intermediate lift-off lengths with regards to the other schemes) and, hence, it cannot be inferred that shorter ID implies shorter LOL when comparing among chemical mechanisms as a consequence of the different thermochemical paths predicted by the oxidation schemes.

Additionally, it is observed that in the oxygen sweep the calculations show a slight loss of sensitivity to the oxygen concentration for the LOL as seen in the case of $X_{O_{2}}=0.21$, predicting all the mechanisms a similar value except for Cai, which even predicting longer LOL, provides a good parallelism with experimental results. A similar study on the influence of chemical mechanisms was performed in [53], where the authors compared Wang, Yao and a previous smaller version of the Cai mechanism [54] with a well-mixed (all of them) and a transported PDF (Yao and Cai) approaches. Overestimation in both ignition delay and lift-off length by the Wang mechanism was observed, while Yao with the TPDF approach showed the most accurate predictions. Both observations are consistent with the present work. The smaller version of Cai mechanism showed similar results to Yao, with a better sensitivity of lift-off length to oxygen concentration in agreement with the current work.

Finally, figure 11 shows LOL as a function of ID for experimental and modelled results for the three sweeps. It is evidenced that reducing ID implies a reduction of LOL 13 , and this fact suggests that chemistry has an important role in the ignition process as well as in the location of the flame base. This argument has been advocated in the literature in order to argue that auto-ignition may be one of the flame stabilization mechanism in diesel sprays [55, 56.

\subsection{Description of the spray auto-ignition process}

This section describes spray A auto-ignition in terms of mass species and heat release evolutions comparing the solutions provided by the different mechanisms and analysing the impact of the boundary conditions. 


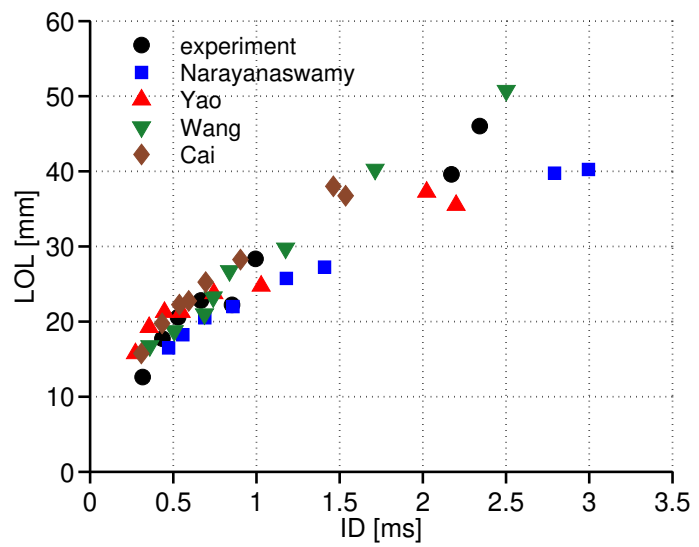

Figure 11: Relationship between ID and LOL for experiments and simulations.

Figure 12 shows the temporal species mass evolution and heat release integrated in the whole cylinder for the nominal case and the four mechanisms. Similar to figure 4 the species are multiplied by a factor in order to share the same scale. Again species representative of the initial, intermediate and final products $\left(\mathrm{CH}_{2} \mathrm{O}, \mathrm{CO}, \mathrm{CO}_{2}\right)$ as well as a soot precursor $\left(\mathrm{C}_{2} \mathrm{H}_{2}\right)$, chemically relevant for soot predictions, are included. 

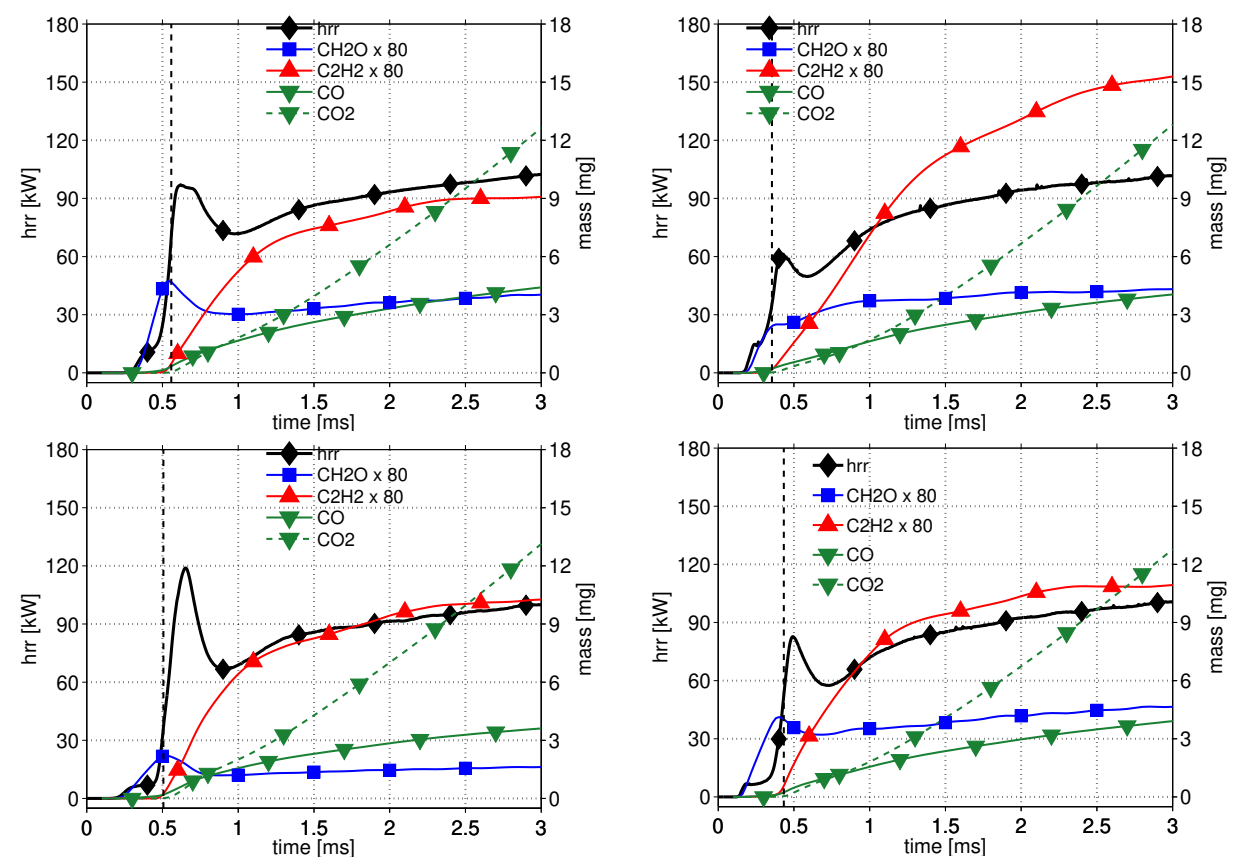

Figure 12: Temporal species mass and heat release evolutions for the turbulent spray for the nominal case. Top: Narayanaswamy (left), Yao (right), bottom: Wang (left) and Cai (right) mechanisms. Vertical dashed line corresponds to the ignition delay for each mechanism.

Heat release in the premixed phase is clearly different for Yao mechanism, which due to the very early ignition has a small peak in this phase, while Narayanaswamy and Cai schemes show similar profiles and Wang predicts a very intense peak.

$\mathrm{CH}_{2} \mathrm{O}$ is generated during the start of combustion, therefore, it appears during the premixed phase and rapidly stabilizes while intermediate and final species, such as $\mathrm{CO}$ and $\mathrm{CO}_{2}$, are generated during the diffusion phase. As will be seen later, when the flame is stabilized, $\mathrm{CH}_{2} \mathrm{O}$ is found in the partially premixed combustion zone while other species, such as $\mathrm{CO}$ and $\mathrm{CO}_{2}$, which appear at more advanced stages of the combustion, are observed in the diffusion flame. 
The previous figure shows that species produced during the start of combustion and, consequently, temporally during the premixed phase and spatially in the region of the partially premixed combustion region (close to the LOL), are observed to be clearly affected by the chemical scheme. This is in line with figure ${ }_{585}^{4}$ where it was shown that the low temperature stage shows the more evident differences among chemical schemes. On the contrary, the analysed mechanisms predict similar evolutions for the species related to the high temperature stage, which is related to the temporal diffusion phase and the spatial diffusion flame.

Concerning soot formation, soot precursor $\mathrm{C}_{2} \mathrm{H}_{2}$ is clearly affected by the oxidation scheme, with Yao mechanism producing the highest values and, hence, yielding presumably the largest soot mass among all of them.

Regarding the effect of the boundary conditions on the heat release and the species mass evolution, figures 13 and 14 gather the results for the temperature (with $X_{\mathrm{O}_{2}}=0.15$ ) and oxygen sweeps (with $T_{a m b}=900 \mathrm{~K}$ ), respectively, for 595 Narayanaswamy mechanism. 

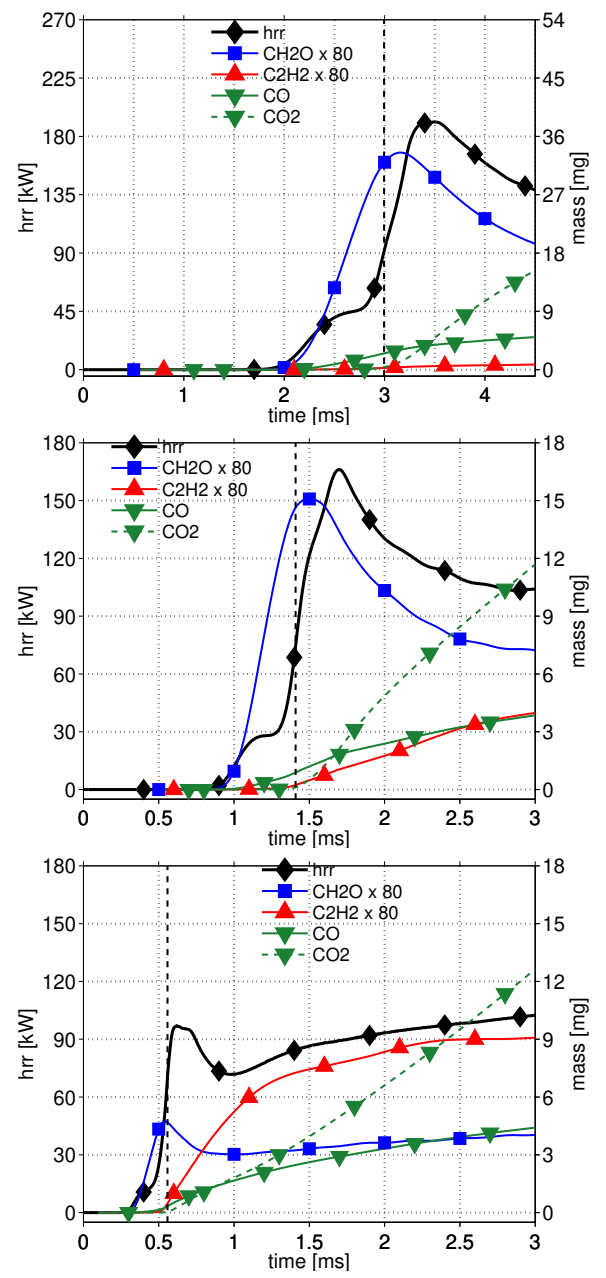

Figure 13: Temporal species mass and heat release evolutions for the turbulent spray. Temperature sweep: $X_{\mathrm{O}_{2}}=0.15$ and $T_{a m b}=750$ (top), 800 (centre) and 900 (bottom) K. Vertical dashed line corresponds to the ignition delay for each case. Scales are common for all the figures except for the $750 \mathrm{~K}$ case. Calculations performed for Narayanaswamy mechanism. 

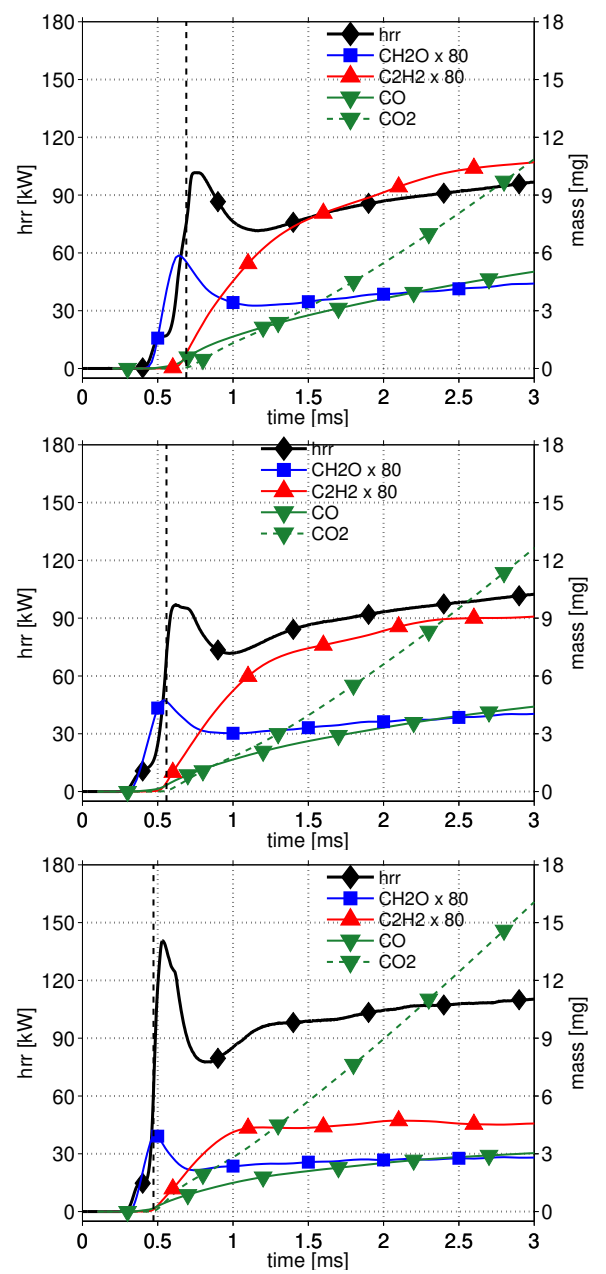

Figure 14: Temporal species mass and heat release evolutions for the turbulent spray. Oxygen sweep: $T_{a m b}=900 \mathrm{~K}$ and $X_{O_{2}}=0.13$ (top), 0.15 (centre) and 0.21 (bottom). Vertical dashed line corresponds to the ignition delay for each case. Calculations performed for Narayanaswamy mechanism.

Figure 13 confirms that reducing the ambient temperature decreases $\mathrm{C}_{2} \mathrm{H}_{2}$ mass, being almost negligible for the $750 \mathrm{~K}$ case of the temperature sweep. This suggests that this would be a non-sooting flame [57, 58, 3]. This effect is due to the displacement of the combustion region to leaner mixtures due to

the lift-off length increase that avoids soot formation [59], as will be shown in 
next section. Besides, due to the reduction of air entrainment prior to ignition, the premixed burn is decreased in magnitude and duration when increasing the ambient temperature [60].

Figure 14 shows that increasing oxygen concentration produces less $\mathrm{C}_{2} \mathrm{H}_{2}$ consequence of such symmetry. 

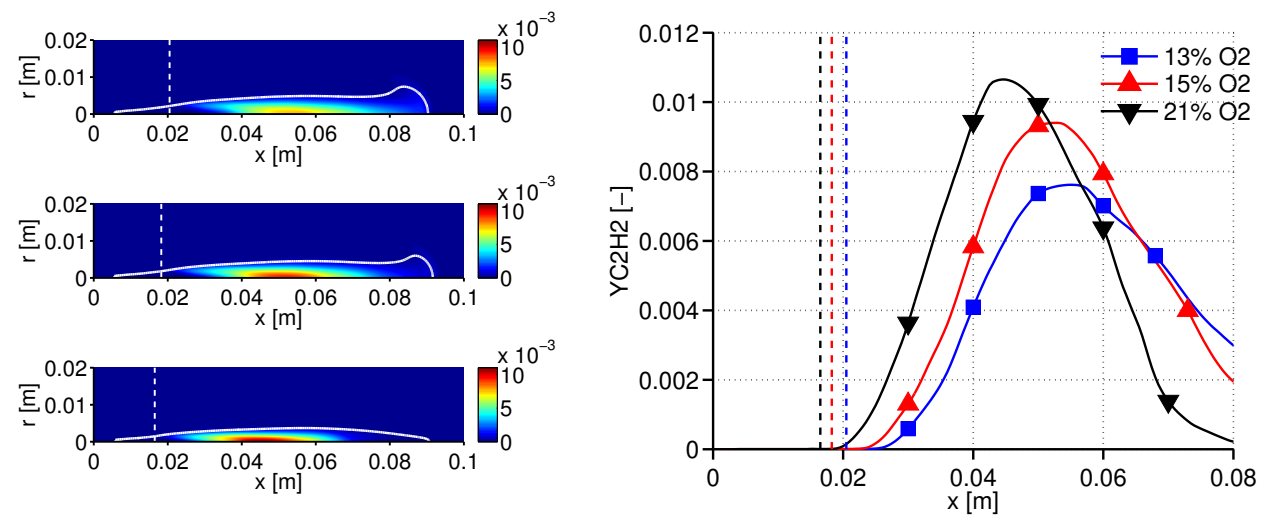

Figure 15: Left figure: $\mathrm{C}_{2} \mathrm{H}_{2}$ mass fraction fields for the oxygen concentration sweep with $\mathrm{X}_{\mathrm{O}_{2}}=$ 0.13 (top), 0.15 (center) and 0.21 (bottom). White solid line corresponds to the stoichiometric mixture fraction level curve and white dashed line indicates modelled LOL position. Right figure: $\mathrm{C}_{2} \mathrm{H}_{2}$ mass fraction on the axis for the oxygen parametric variation. Dashed line indicates modelled lift-off length position for each case. Results for very advanced instants and Narayanaswamy mechanism.

Moreover, from figure 14 it arises that increasing oxygen concentration may lead to a more intense premixed combustion in spite of a shorter ignition delay and, hence, a lower injected fuel mass at ignition. This behaviour has been confirmed experimentally [59].

Finally, although not shown here, spray ignition is observed to start at slightly lean mixtures in the early instants due to their high initial adiabatic mixing temperature [7]. This is in agreement with the reduced $\mathrm{ID}_{1}$ values for lean mixtures observed in figure 2 that caused first ignition kernels to appear at such mixtures in flamelet calculations too (see figures 5, 6 and 7). The ignition in lean mixtures promotes in turn the combustion to richer mixtures due to the intense mixing induced by the turbulent flow [50, 17] where combustion at high temperature propagates. This behaviour has been experimentally observed [7] and LES simulations corroborate this evolution too [62]. The same observation was obtained from the flamelet calculations and, as expected, is retained for the spray. 


\subsection{Analysis of the spray flame structure in quasi-steady regime}

In this section spray flame structure during the so-called quasi-steady regime, corresponding to instants around $3 \mathrm{~ms}$ or more advanced, is thoroughly analysed from different points of view with very special attention to those representations based on combustion variables.

First, the flame structure is described in terms of the progress variable chemical source term, $\partial \widetilde{Y}_{c} / \partial t$, which is the average value from the transient term of equation 1 and is extremely useful because it traces the whole combustion process and shows where reactions releasing heat occur as pointed out in section 3.1 . Figure 16 represents the spatial field for this variable at an advanced instant for the nominal case with Narayanaswamy mechanism. Logarithmic scale is used due to the wide range values in order to better visualize the whole structure of the flame.

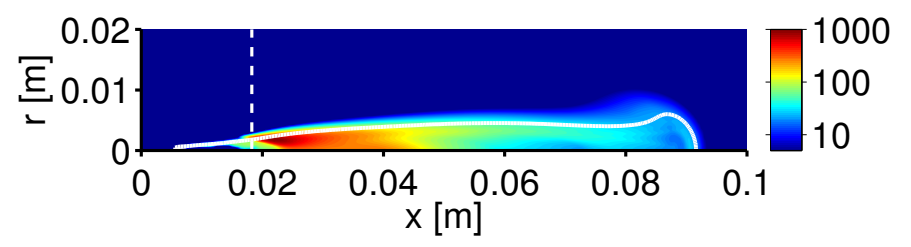

Figure 16: $\partial \widetilde{Y}_{c} / \partial t$ spatial field at an advanced instant for the nominal case with Narayanaswamy mechanism. A logarithmic scale is used for $\partial \widetilde{Y}_{c} / \partial t$. White solid line corresponds to the stoichiometric mixture fraction level curve and white dashed line indicates simulated LOL position.

The $\partial \widetilde{Y}_{c} / \partial t$ spatial field reveals that the flame structure is composed of a partially premixed combustion region positioned downstream of the lift-off length which shows a vigorous combustion as deduced from the high burning rate. The location of the most intense chemical source term for this partially premixed combustion is radially displaced from the axis in the rich mixture regions (around $\widetilde{\phi}=1.7$ ) as will be seen in more detail in subsequent figures.

Finally, a diffusion flame is established on the stoichiometric surface with a burning rate that is clearly less intense than in the partially premixed combustion zone $\left(\partial \widetilde{Y}_{c} / \partial t\right.$ falls one or two orders of magnitude). Note that the diffusion flame is not only limited to the average stoichiometric level curve due to the tur- 
bulent fluctuations retained in the model. These fluctuations are accounted for by means of presumed PDFs for both the mixture fraction and the scalar dissipation rate variables (as explained in section 2.1) providing, hence, a distributed turbulent flame brush, as expected in the RANS context [13].

The flame structure shown in figure 16 matches the picture suggested by Dec [8] for diesel flames and this is considered a qualitative validation of the performance of the model. This is complemented when representing the species in the $Z-T$ map, where species belonging to the low temperature chemistry, such as $\mathrm{CH}_{2} \mathrm{O}$, are located at rich mixtures and low temperatures while tracers of the high temperature stage, like $\mathrm{OH}$, only extend around the stoichiometric mixture and high temperatures. In order to describe in more detail the flame structure, Supplementary Material (a) contains the species location represented in the $Z-T$ map as well as the effect of the chemical mechanism and the boundary conditions in determining the reactive mixture fractions.

The analysis is closed providing a deeper insight of the flame structure in the $Z-T$ map representation. For this purpose, representative level curves in physical space for the most important variables defining combustion are found and the pairs $(Z, T)$ along such level curves are taken and represented in the $Z-T$ map allowing to condensate in a single picture the whole combustion process.

The variables considered for this purpose are $\widetilde{Z^{\prime \prime 2}}$ and $\tilde{\chi}$ as non-reactive scalars, which define the state of the mixture in conjunction with $\widetilde{Z}$, and the chemical source term $\partial \widetilde{Y}_{c} / \partial t$, as a tracer of the reaction zone, which is complemented with temperature. These representations are extremely useful because for this specific model and, more generally, for any flamelet based combustion model, they contain all the information necessary to interpret the combustion since they are build upon the controlling variables of the reactive flow.

Table 2 contains the values related to the different level curves included in figures 17, 19 and 20. To distinguish the different level curves for a given variable markers are used: square is used for the minimum value, circle for the next value and triangle for the maximum value (if a third value is included). 
For each boundary condition the values have been chosen to be representative of the combustion process since it develops at different physical regions.

Table 2: Values of the variables for the represented level curves in figures 17 19 and 20

\begin{tabular}{cccccc}
\hline Variable & $750 \mathrm{~K} 15 \% \mathrm{O} 2$ & $800 \mathrm{~K} 15 \% \mathrm{O} 2$ & $900 \mathrm{~K} 15 \% \mathrm{O} 2$ & $900 \mathrm{~K} 13 \% \mathrm{O} 2$ & $900 \mathrm{~K} 21 \% \mathrm{O} 2$ \\
\hline \hline$\widetilde{Z}^{\prime \prime 2} \times 10^{4}$ & $1.3,2$ & $3.3,4.5,5.5$ & $7,10,14$ & $5,7,9$ & $12,18,25$ \\
\hline$\widetilde{\chi}(1 / \mathrm{s})$ & $0.3,0.4,0.8$ & $2.5,4$ & $3,20,30$ & $3,10,20$ & $10,50,80$ \\
\hline$\partial \widetilde{Y}_{c} / \partial t(1 / \mathrm{s})$ & 150,350 & 200,500 & $300,600,900$ & 300,600 & 600,1500 \\
\hline
\end{tabular}

The representations of the level curves in the $Z-T$ maps are divided in two 695 figures. The first one contains the level curves for $\widetilde{Z^{\prime \prime 2}}$ and $\widetilde{\chi}$ while in a second figure the level curves for $\partial \widetilde{Y}_{c} / \partial t$ are represented. Plots in figure 17 contain this representation for the nominal case with all the mechanisms at advanced instants. The initial adiabatic mixing profile between air and fuel as well as the upper contour of the map are included with black dashed lines. Besides, the stoichiometric level curve is denoted by a vertical black dashed line. 

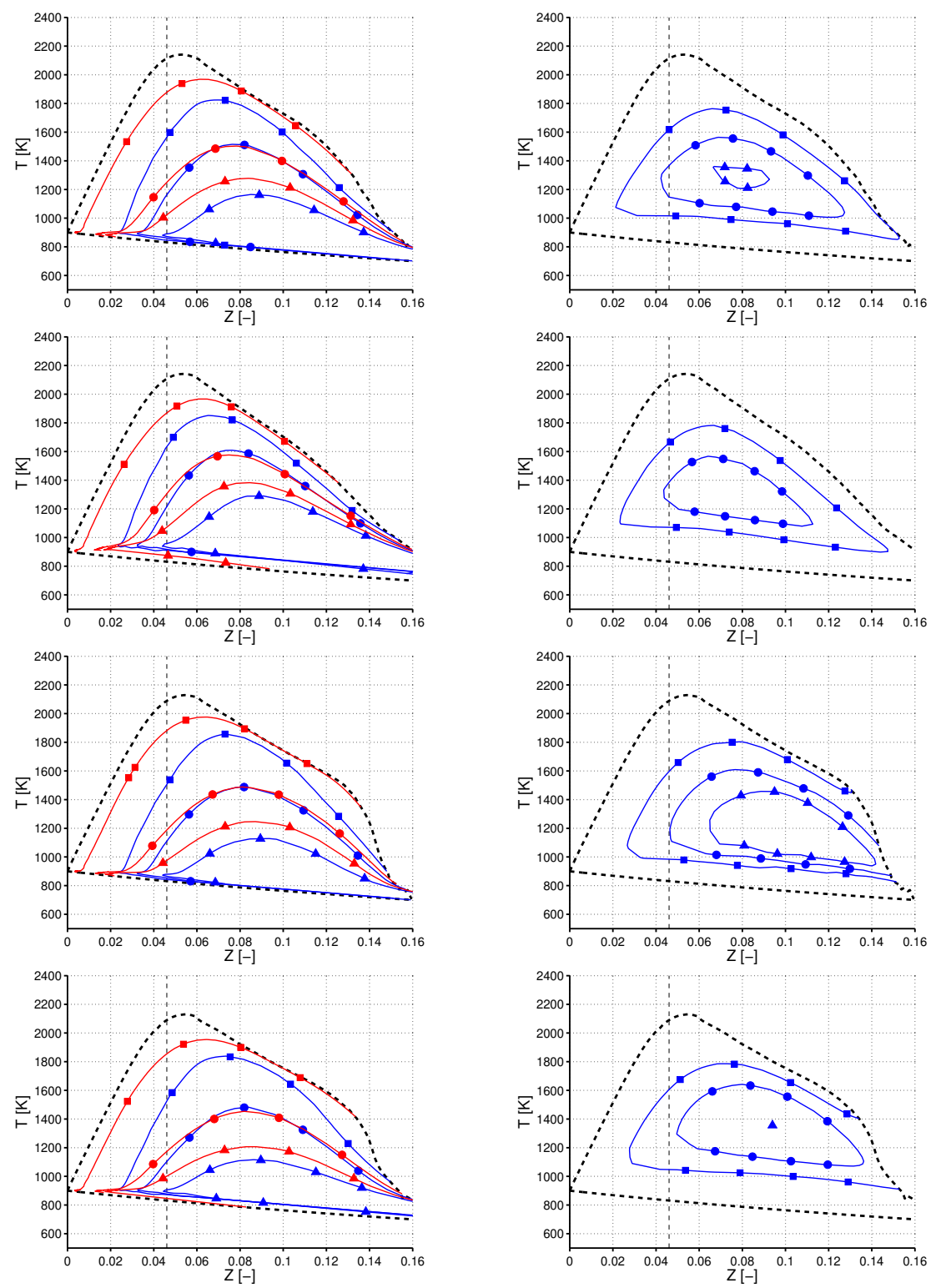

Figure 17: $Z-T$ maps with level curves for $\widetilde{Z^{\prime \prime 2}}$ (blue) and $\widetilde{\chi}$ (red) (left column) and $\partial \widetilde{Y}_{c} / \partial t$ (right column) for the nominal case with Narayanaswamy (top row), Yao (second row), Wang (third row) and Cai (last row). Markers distinguish between different level curve values (table 2 . Black dashed line corresponds to the initial adiabatic mixing line and the upper contour of the map. Vertical black dashed line indicates the stoichiometric mixture fraction.

As seen from figure 17, the source term is mainly due to the partially pre- 
mixed combustion zone (see figure 16), which appears in a broad region of mixture fractions and temperatures, compared to the diffusion flame, which is less intense and therefore results in lower source terms (see figure 16). The latter one is actually very close to the highest temperatures, that is, to the upper contour of the map. $\partial \widetilde{Y}_{c} / \partial t$ contours show that reaction rates are especially intense (this depends on the mechanism too) for $\widetilde{Z}$ between 0.07 and 0.09 (corresponding to $\widetilde{\phi}$ between 1.5 and 2) and temperatures that range between 1200 and $1400 \mathrm{~K}$, in agreement with [8]. In this zone $\widetilde{Z^{\prime \prime 2}}$ shows values around $1.2 \cdot 10^{-3}$ and $\tilde{\chi}$ around $251 / \mathrm{s}$ (that, in this case, corresponds to $\tilde{\chi}_{s t}=251 / \mathrm{s}$ ).

More specifically, this means that the partially premixed combustion develops mainly in a rich mixture region with the instantaneous mixture fraction ranging mainly in the interval $0.07 \pm 0.035$ (taking $\widetilde{Z^{\prime \prime 2}}=1.2 \cdot 10^{-3}$ ) and a flame with $\widetilde{\chi}_{s t}=251 / \mathrm{s}$. This is a moderate value for a flame scalar dissipation rate considering that flamelet auto-ignition range extends until $\chi_{s t}=1061 / \mathrm{s}$, approximately. Consequently, the partially premixed combustion zone is presumably not strongly affected by extinction effects and, hence, this result confirms that the diesel reactive spray is properly modelled using a thermochemical database generated from flamelets belonging to the auto-ignition range [25].

In order to deepen in the previous statement, figure 18 shows the log-normal $\mathrm{PDF}$ for $\widetilde{\chi}_{s t}=251 / \mathrm{s}$ and $\sigma=\sqrt{2}$, used in the generation of the turbulent combustion manifold. It is clearly seen that the area enclosed by the PDF for $\chi_{s t}$ values greater than $106 \mathrm{1} / \mathrm{s}$ is marginal and the probability of reaching such zone is only $4.16 \%$. This means that the probability of reaching extinction conditions in the partially premixed combustion zone is extremely low and, hence, extinction effects are not of first order.

The different mechanisms show some differences in the position and the values taken in the region of the partially premixed combustion region. Yao and Narayanaswamy schemes predict such region positioned in similar temperature and mixture fraction values. However, Cai and especially Wang mechanisms show a very high chemical source term that is displaced to very rich mixtures in the same way that flamelet calculations (figures 5, 6 and 77) showed that ignition 


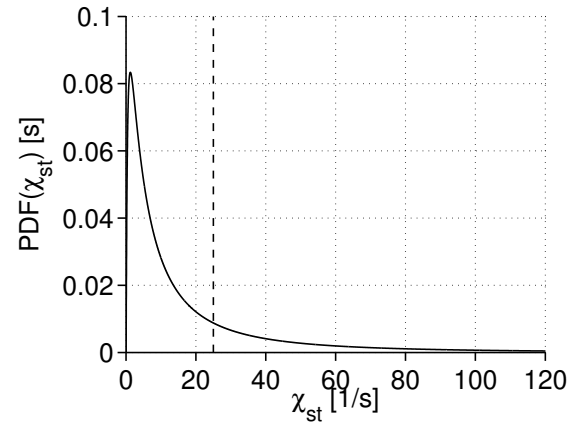

Figure 18: Log-normal PDF defined by $\tilde{\chi}_{s t}=251 / \mathrm{s}$ and $\sigma=\sqrt{2}$. Black vertical dashed line indicates $\tilde{\chi}_{s t}=251 / \mathrm{s}$.

occurred at richer mixtures than those computed with the other mechanisms. This behaviour was explained as a consequence of the homogeneous reactors ignition delays from figure 2 and the extremely high chemical terms observed during the second combustion stage (figure 3). As described in Supplementary Material (a) the profile of the upper contour in the region that delimits reactive and non-reactive mixtures for Wang scheme is sharp in the same way that sharp profiles were observed in this interface for its flamelet profiles (see figure 5). In general, similar observations can be applied to the other schemes.

Regarding the non-reactive scalars, they take similar values in the whole map for all the mechanisms since the differences in density predicted by the chemical schemes are not so high to provoke noticeable discrepancies in the mixing variables.

Finally, figures 19, on the one hand, and 20, on the other hand, gather the impact of the boundary conditions on the represented flame variables for the temperature (with $X_{O_{2}}=0.15$ ) and oxygen (with $T_{a m b}=900 \mathrm{~K}$ ) sweeps calculated with Narayanaswamy mechanism. Again the maps correspond to advanced instants. 

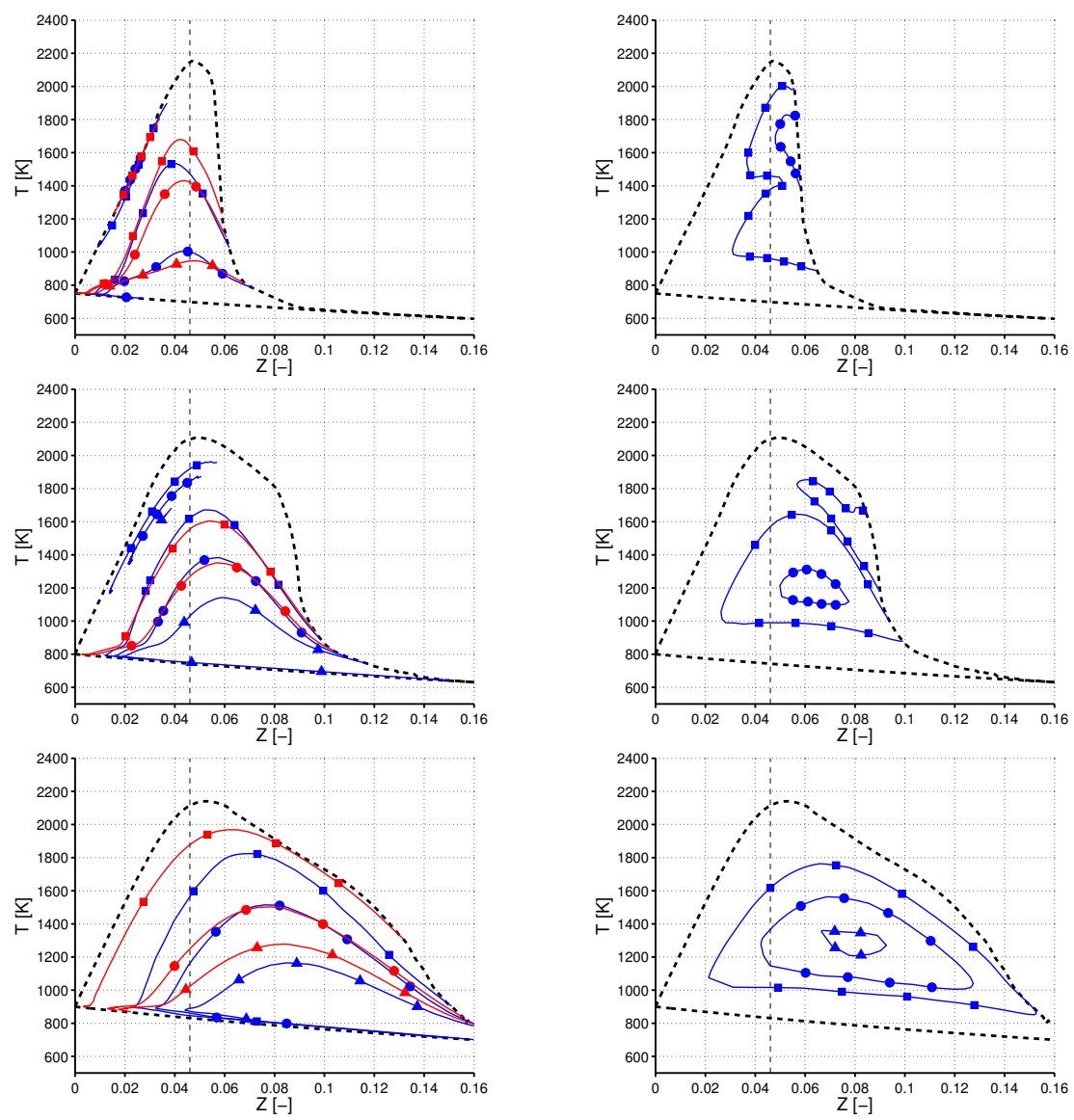

Figure 19: $Z-T$ maps with level curves for $\widetilde{Z^{\prime \prime 2}}$ (blue) and $\widetilde{\chi}$ (red) (left column) and $\partial \widetilde{Y}_{c} / \partial t$ (right column). Temperature sweep: $X_{O_{2}}=0.15$ and $T_{a m b}=750$ (top), 800 (centre) and 900 (bottom) K. Markers distinguish between different level curve values (table 2f. Black dashed line corresponds to the initial adiabatic mixing line and the upper contour of the map. Vertical black dashed line indicates the stoichiometric mixture fraction. Calculations performed with Narayanaswamy mechanism. 

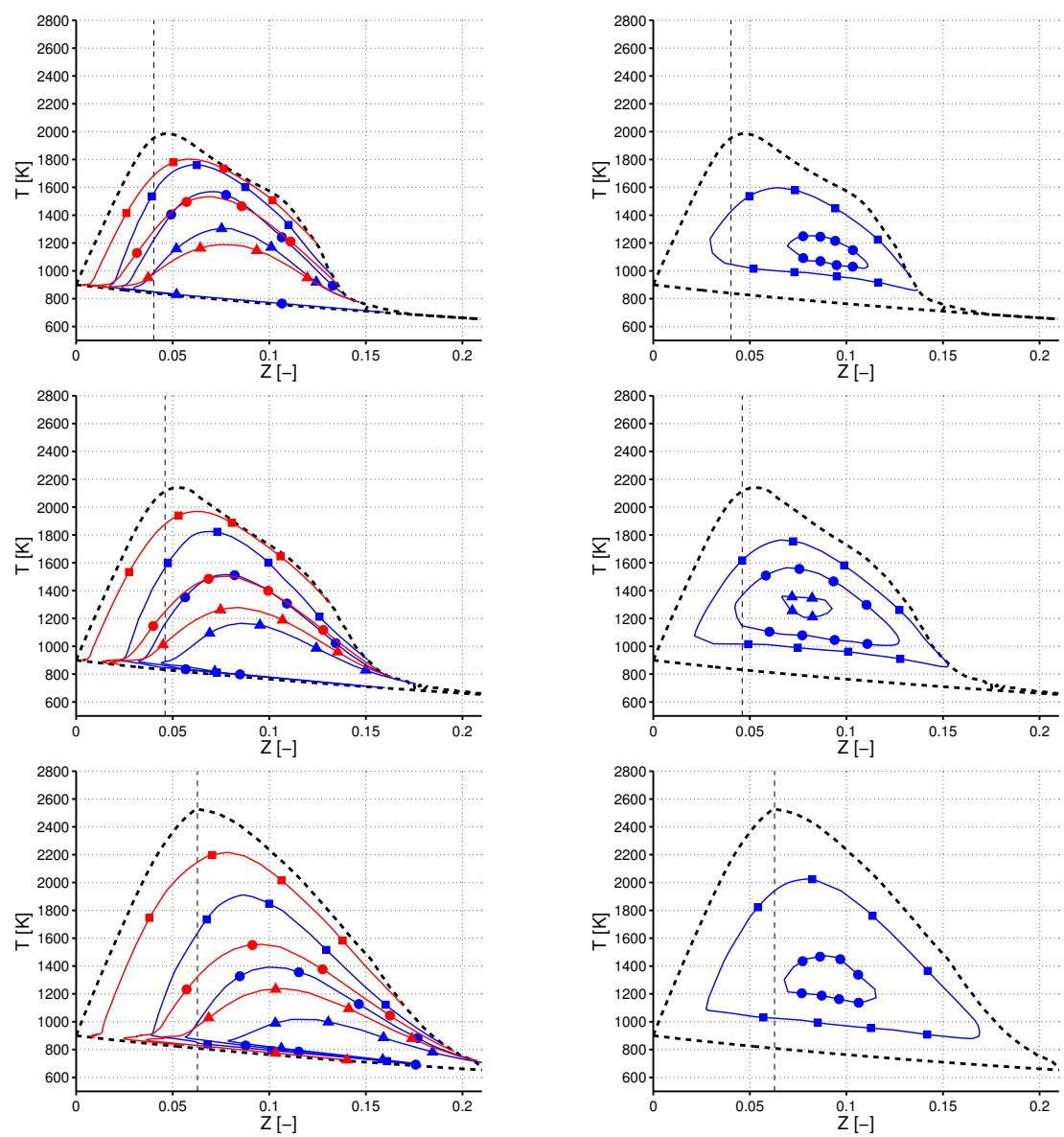

Figure 20: $Z-T$ maps with level curves for $\widetilde{Z^{\prime \prime 2}}$ (blue) and $\tilde{\chi}$ (red) (left column) and $\partial \widetilde{Y}_{c} / \partial t$ (right column). Oxygen sweep: $T_{a m b}=900 \mathrm{~K}$ and $X_{O_{2}}=0.13$ (top), 0.15 (centre) and 0.21 (bottom). Markers distinguish between different level curve values (table 2). Black dashed line corresponds to the initial adiabatic mixing line and the upper contour of the map. Vertical black dashed line indicates the stoichiometric mixture fraction. Calculations performed with Narayanaswamy mechanism.

Values appearing in table 2 are considered representative for these cases and, thus, they give a preliminary idea of how the boundary conditions impact on the controlling variables where combustion is sustained, revealing that the partially premixed combustion region is always placed at low/intermediate levels of $\widetilde{Z^{\prime \prime 2}}$ and $\tilde{\chi}$. This is in agreement with results in the literature where it has been verified that combustion adapts to the local flow dynamics in order to position 
at low/intermediate values of the mixing turbulent variables [49].

Figure 19 evidences that increasing the reactivity by means of the ambient temperature displaces the partially premixed combustion zone to richer mixtures. However, the change in oxygen concentration has only a mild influence on the position of the mixtures with the highest reactivity (although a slight enrichment is observed when decreasing the oxygen concentration). This stems from the analysis of the ignition for the flamelets where it was revealed that $Z_{M R}$ became higher when increasing the ambient temperature but did not almost change when modifying the oxygen concentration. As a consequence, the partially premixed combustion occurs at leaner equivalence ratios when increasing the oxygen concentration.

Furthermore, there is a clear increase of the values of the non-reactive scalars in the region of the partially premixed combustion when augmenting the ambient temperature but this change is more moderate when reducing the EGR. 770 This is due to the strong increase of the lift-off length when changing the ambient temperature but the moderate change of this distance when varying the oxygen concentration. Summarizing, the increase of reactivity of the mixture, by ambient temperature or oxygen concentration, enables to sustain combustion in regions of more intense convection and diffusion.

775 In addition, for low temperature cases a second peak of the partially premixed combustion zone is observed for $\partial \widetilde{Y}_{c} / \partial t$ for very rich mixtures and high temperatures (around $1600 \mathrm{~K}$ ), as observed in figure 19 for the cases of 750 and $800 \mathrm{~K}$. In the spatial representation this peak is found on the axis and, hence, the partially premixed combustion zone is composed of two regions of high chemical reactivity: a region of moderate rich mixtures radially displaced from the axis and a region positioned on the axis at richer mixtures and higher temperatures. When increasing the reactivity of the mixture this second peak disappears. This points that the structure of the of low reactivity diesel sprays may be more complex and, hence, requires further analysis.

785

Finally, an additional observation is that it seems that the level curves for $\widetilde{Z^{\prime \prime 2}}$ and $\tilde{\chi}$ are approximately overlapped for all the boundary conditions. Some 
comments about this behaviour are given in Supplementary Material (b).

\section{Conclusions}

The influence of the mechanism choice and the boundary conditions have been analysed thoroughly in order to shed light on their impact on spray A structure as a representative spray for modern diesel engines. An advanced combustion model based on the flamelet concept has been used for this purpose which has enabled to figure out the effect of the oxidation scheme as well as the boundary conditions at different stages of complexity. In this sense, a systematic analysis has been performed which extends from homogeneous reactors, where only chemistry is considered, to CFD, where coupled physical and chemical phenomena interact.

Along this work several conclusions have emerged which have been corroborated at the different stages of the analysis from different points of view and have been connected with experimental and modelling studies. The agreement between these works and the current analysis supports the suitability of the flamelet based models and, more particularly, the ADF model, to be applied to the simulation of reacting sprays in the frame of diesel engines.

The main conclusions are briefly summarized in the following lines:

- The mechanism choice has a strong influence on the low temperature chemistry and the soot precursors prediction at all the calculation stages. The high temperature chemistry is barely affected by the chemical scheme.

- The different thermochemical evolution and reactivity between mixtures at low temperature may modify the evolution of the whole combustion flame when diffusion is introduced (flamelets) when comparing between mechanisms.

- The latency period between $\mathrm{ID}_{1}$ and $\mathrm{ID}_{2}$ and how the chemical source term develops during the mixtures ignition are relevant in order to understand how combustion develops for the different mixture fractions when diffusion 
effects are introduced. This aspect is deeply related to the sharpness of the flamelet temporal profiles and, consequently, the diffusion fluxes intensity.

- All the analysed mechanisms predict (at all the stages) an ignition starting at lean mixtures which is rapidly displaced to rich mixtures. This displacement is enhanced with the increase of the diffusion effects (increase of strain rate).

- The model reproduces the ignition delay and lift-off length trends. Lower ignition delays do not necessarily imply lower lift-off lengths when comparing between mechanisms (once the boundary conditions are fixed) due to the different thermochemical paths, especially those related to low temperature chemistry.

- Reducing the reactivity of the mixture by means of the ambient temperature displaces combustion to leaner mixture fractions while combustion location is slightly enriched with the decrease of the oxygen concentration. With regards to the soot precursors predictions, their production is decreased when lowering the ambient temperature or augmenting the oxygen concentration in agreement with experimental findings.

- The partially premixed combustion is located at rich mixtures and intermediate temperatures with moderate values for $\widetilde{Z^{\prime \prime 2}}$ and $\widetilde{\chi}$. There exists a remarkable dependence of these variables on the boundary conditions, especially on the ambient temperature for which a variation of one order of magnitude for $\widetilde{Z^{\prime \prime 2}}$ and $\widetilde{\chi}$ is found.

Finally, referring to the issue posed at the introduction about the influence of the chemical mechanism on the turbulent reacting spray, which was one of the motivations of this work, the previous conclusions reveal that most of the differences observed in homogeneous reactors calculations persist at all the levels (homogeneous reactors, flamelets and CFD) with a special impact on the structure of the partially premixed combustion zone in the turbulent reacting spray. 


\section{Acknowledgments}

845 Authors acknowledge that this work was possible thanks to the Ayuda para la Formación de Profesorado Universitario (FPU 14/03278) belonging to the Subprogramas de Formación y de Movilidad from the Ministerio de Educación, Cultura y Deporte from Spain. This work was partially funded by the Government of Spain through CHEST Project (TRA2017-89139-C2-1-R).

\section{References}

[1] T. W. Ryan, T. J. Callahan, Homogeneous charge compression ignition of diesel fuel, Technical Report, SAE Technical Paper, 1996.

[2] L. M. Pickett, G. Bruneaux, R. Payri, Sandia National Laboratories, Livermore, CA, https://ecn.sandia.gov/ (2018).

[3] N. Maes, M. Meijer, N. Dam, B. Somers, H. B. Toda, G. Bruneaux, S. A. Skeen, L. M. Pickett, J. Manin, Combustion and Flame 174 (2016) 138-51.

[4] M. Bardi, R. Payri, L. M. Malbec, G. Bruneaux, L. M. Pickett, J. Manin, T. Bazyn, C. L. Genzale, Atomization and Sprays 22 (2012).

[5] J. Benajes, R. Payri, M. Bardi, P. Martí-Aldaraví, Applied Thermal Engineering 58 (2013) 554-63.

[6] R. Payri, J. M. García-Oliver, T. Xuan, M. Bardi, Applied Thermal Engineering 90 (2015) 619-29.

[7] S. A. Skeen, J. Manin, L. M. Pickett, Proceedings of the Combustion Institute 35 (2015) 3167-74.

865

[8] J. E. Dec, A conceptual model of DI diesel combustion based on laser-sheet imaging, Technical Report, SAE Technical Paper, 1997.

[9] K. Narayanaswamy, P. Pepiot, H. Pitsch, Combustion and Flame 161 (2014) 866-84. 
[10] T. Yao, Y. Pei, B. J. Zhong, S. Som, T. Lu, in: 9th US National Combustion Meeting, Cincinnati, Ohio, pp. 17-20.

[11] T. Poinsot, D. Veynante, Theoretical and numerical combustion, RT Edwards Inc., 2005.

[12] N. Peters, Turbulent combustion, Cambridge University Press, 2000.

[13] S. Bhattacharjee, D. C. Haworth, Combustion and Flame 160 (2013) 2083102.

[14] Y. M. Wright, O. N. Margari, K. Boulouchos, G. De Paola, E. Mastorakos, Flow, Turbulence and Combustion 84 (2010) 49-78.

[15] M. Bolla, D. Farrace, Y. M. Wright, K. Boulouchos, E. Mastorakos, Combustion Theory and Modelling 18 (2014) 330-60.

[16] Y. Pei, E. R. Hawkes, S. Kook, G. M. Goldin, T. Lu, Combustion and Flame 162 (2015) 2006-19.

[17] Y. Pei, E. R. Hawkes, M. Bolla, S. Kook, G. M. Goldin, Y. Yang, S. B. Pope, S. Som, Combustion and Flame 168 (2016) 420-35.

[18] N. Peters, Progress in Energy and Combustion Science 10 (1984) 319-39.

[19] O. Gicquel, N. Darabiha, D. Thévenin, Proceedings of the Combustion Institute 28 (2000) 1901-8.

[20] J. A. van Oijen, L. P. H. de Goey, Combustion Science and Technology 161 (2000) 113-37.

[21] L. P. H. de Goey, J. A. van Oijen, H. Bongers, G. R. A. Groot, in: European Combustion Meeting, Orléans (France).

[22] J. B. Michel, O. Colin, D. Veynante, Combustion and Flame 152 (2008) 80-99.

[23] J. B. Michel, O. Colin, D. Veynante, Flow, Turbulence and Combustion 83 (2009) 33-60. 
[35] J. M. Desantes, J. M. Garcia-Oliver, J. M. Pastor, A. Pandal, Atomization and Sprays 26 (2016).

[36] J. K. Dukowicz, Journal of computational Physics 35 (1980) 229-53.

[37] J. Tillou, J. B. Michel, C. Angelberger, D. Veynante, Combustion and 920

[24] V. Knop, J. B. Michel, O. Colin, Applied Energy 88 (2011) 4968-79.

[25] J. B. Michel, O. Colin, International Journal of Engine Research (2013) 1468087413488590 .

[26] R. Novella, A. García, J. M. Pastor, V. Domenech, Mathematical and Computer Modelling 54 (2011) 1706-19.

[27] A. Frassoldati, G. D'Errico, T. Lucchini, A. Stagni, A. Cuoci, T. Faravelli, A. Onorati, E. Ranzi, Combustion and Flame 162 (2015) 3991-4007.

[28] J. M. Desantes, J. J. López, J. M. García-Oliver, D. López-Pintor, Combustion and Flame 182 (2017) 76-89.

[29] B. Naud, R. Novella, J. M. Pastor, J. F. Winklinger, Combustion and Flame 162 (2015) 893-906.

[30] D. Aubagnac-Karkar, J.-B. Michel, O. Colin, P. E. Vervisch-Kljakic, N. Darabiha, Combustion and Flame 162 (2015) 3081-99.

[31] A. Varna, M. J. Cleary, E. R. Hawkes, Combustion and Flame 181 (2017) 354-64.

[32] J. M. Desantes, J. M. García-Oliver, R. Novella, E. J. Pérez-Sánchez, Applied Thermal Engineering 117 (2017) 50-64.

[33] F. Payri, J. M. García-Oliver, R. Novella, E. J. Pérez-Sánchez, in: ILASS Europe. 28th European Conference on Liquid Atomization and Spray Systems, Valencia, Spain, pp. 678-85.

34] OpenFOAM, http://www.openfoam.com/ (2017).

Flame 161 (2014) 525-40. 
[38] F. Payri, R. Novella, J. M. Pastor, E. J. Pérez-Sánchez, Applied Mathematical Modelling 49 (2017) 354-74.

[39] B. Fiorina, O. Gicquel, L. Vervisch, S. Carpentier, N. Darabiha, Combustion and Flame 140 (2005) 147-60.

[47] A. L. Kastengren, F. Z. Tilocco, C. F. Powell, J. Manin, L. M. Pickett, R. Payri, T. Bazyn, Atomization and Sprays 22 (2012) 1011-52.

[48] S. S. Vasu, D. F. Davidson, Z. Hong, V. Vasudevan, R. K. Hanson, Proceedings of the Combustion Institute 32 (2009) 173-80.

[49] M. Epaminondas, Progress in Energy and Combustion Science 35 (2009) 945

[50] C. Gong, M. Jangi, X. S. Bai, Applied Energy 136 (2014) 373-81. 
[51] R. N. Dahms, G. A. Paczko, S. A. Skeen, L. M. Pickett, Proceedings of the Combustion Institute 36 (2017) 2615-23.

[52] H. P. S. Shen, J. Steinberg, J. Vanderover, M. A. Oehlschlaeger, Energy \& Fuels 23 (2009) 2482-9.

[53] M. A. Chishty, M. Bolla, E. R. Hawkes, Y. Pei, S. Kook, Combustion and Flame 192 (2018) 101-19.

[54] M. Davidovic, M. Bode, T. Falkenstein, L. Cai, H. Pitsch, in: LES for Internal Combustion Engine Flows LES4ICE, France.

955 [55] L. M. Pickett, D. L. Siebers, C. A. Idicheria, Relationship between ignition processes and the lift-off length of diesel fuel jets, Technical Report, SAE Technical Paper, 2005.

[56] Y. Pei, M. J. Davis, L. M. Pickett, S. Som, Combustion and Flame 162 (2015) 2337-47.

[57] L. M. Pickett, D. L. Siebers, Combustion and Flame 138 (2004) 114-35.

[58] L. M. Pickett, D. L. Siebers, Non-sooting, low flame temperature mixingcontrolled DI diesel combustion, Technical Report, SAE Technical Paper, 2004.

[59] C. A. Idicheria, L. M. Pickett, Proceedings of the Combustion Institute 31 (2007) 2931-8.

[60] B. Higgins, D. L. Siebers, A. Aradi, Diesel-spray ignition and premixedburn behavior, Technical Report, SAE Technical Paper, 2000.

[61] C. A. Idicheria, L. M. Pickett, Soot formation in diesel combustion under high-EGR conditions, Technical Report, SAE Technical Paper, 2005.

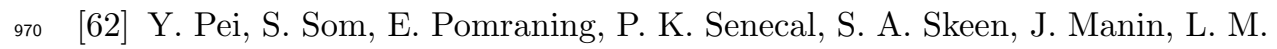
Pickett, Combustion and Flame 162 (2015) 4442-55. 